\title{
Principal Graphs and Manifolds
}

\author{
Alexander Gorban*` and Andrei Zinovyev ${ }^{\# \bullet}$ \\ * Department of Mathematics, University of Leicester \\ University Road, Leicester LE1 7RH, United Kingdom \\ ag153@le.ac.uk \\ \# Institut Curie, 26 rue d'Ulm, Paris 75248, France \\ andrei.zinovyev@curie.fr \\ 'Institute for Computational Modelling Russian Academy of Sciences, \\ Akademgorodok, Krasnoyarsk 660036, Russia
}

\begin{abstract}
In many physical statistical, biological and other investigations it is desirable to approximate a system of points by objects of lower dimension and/or complexity. For this purpose, Karl Pearson invented principal component analysis in 1901 and found 'lines and planes of closest fit to system of points'. The famous $k$-means algorithm solves the approximation problem too, but by finite sets instead of lines and planes. This chapter gives a brief practical introduction into the methods of construction of general principal objects, i.e. objects embedded in the 'middle' of the multidimensional data set. As a basis, the unifying framework of mean squared distance approximation of finite datasets is selected. Principal graphs and manifolds are constructed as generalisations of principal components and $k$-means principal points. For this purpose, the family of expectation/maximisation algorithms with nearest generalisations is presented. Construction of principal graphs with controlled complexity is based on the graph grammar approach.
\end{abstract}

\section{INTRODUCTION}

In many fields of science one meets with multivariate (multidimensional) distributions of vectors representing some observations. These distributions are often difficult to analyse and make sense of due to the very nature of human brain which is able to visually manipulate only with the objects of dimension no more than three.

This makes actual the problem of approximating the multidimensional vector distributions by objects of lower dimension and/or complexity while retaining the most important information and structures contained in the initial full and complex data point cloud.

The most trivial such approximation is collapsing the whole set of vectors into its mean point. The mean point represents the 'most typical' properties of the system, completely forgetting variability of observations. 
The notion of the mean point can be generalized for approximating data by more complex types of objects. In 1901 Pearson proposed to approximate multivariate distributions by lines and planes (Pearson, 1901). In this way the Principal Component Analysis (PCA) was invented, nowadays a basic statistical tool. Principal lines and planes go through the 'middle' of multivariate data distribution and correspond to the first few modes of the multivariate Gaussian distribution approximating the data.

Starting from 1950s (Steinhaus, 1956; Lloyd, 1957; and MacQueen, 1967), it was proposed to approximate the complex multidimensional dataset by several 'mean' points. Thus k-means algorithm was suggested and nowadays it is one of the most used clustering method in machine learning (see a review presented by $\mathrm{Xu} \&$ Wunsch, 2008)).

Both these directions (PCA and K-Means) were further developed during last decades following two major directions: 1) linear manifolds were generalised for non-linear ones (in simple words, initial lines and planes were bended and twisted), and 2) some links between the 'mean' points were introduced. This led to appearance of several large families of new statistical methods; the most famous from them are Principal Curves, Principal Manifolds and Self-Organising Maps (SOM). It was quickly realized that the objects that are constructed by these methods are tightly connected theoretically. This observation allows now to develop a common framework called "Construction of Principal Objects". The geometrical nature of these objects can be very different but all of them serve as data approximators of controllable complexity. It allows to use them in the tasks of dimension and complexity reduction. In Machine Learning this direction is connected with terms "Unsupervised Learning" and "Manifold Learning".

In this chapter we will overview the major directions in the field of principal objects construction. We will formulate the problem and the classical approaches such as PCA and $k$-means in unifying framework, and show how it is naturally generalised for the Principal Graphs and Manifolds and the most general types of principal objects, Principal Cubic Complexes. We will systematically introduce the most used theorems and algorithms developed in this field.

\section{APPROXIMATIONS OF FINITE DATASETS}

Definition. Dataset is a finite set $X$ of objects representing $N$ multivariate (multidimensional) observations. These objects $\mathbf{x}^{i} \in X, i=1 \ldots N$ are embedded in $\mathbf{R}^{m}$ and in the case of complete data are vectors $\mathbf{x}^{i} \in \mathbf{R}^{m}$. We will also refer to the individual components of $\mathbf{x}^{i}$ as $x_{k}^{i}$ such that $\mathbf{x}^{i}=\left(x_{1}^{i}, x_{2}^{i}, \ldots, x_{m}^{i}\right)$; we can also represent dataset as a data matrix $X=\left\{x_{j}^{i}\right\}$.

Definition. Distance function $\operatorname{dist}(\mathbf{x}, \mathbf{y})$ is defined for any pair of objects $\mathbf{x}, \mathbf{y}$ from $X$ such that three usual axioms are satisfied: $\operatorname{dist}(\mathbf{x}, \mathbf{x})=0, \operatorname{dist}(\mathbf{x}, \mathbf{y})=\operatorname{dist}(\mathbf{y}, \mathbf{x})$, $\operatorname{dist}(\mathbf{x}, \mathbf{y})+\operatorname{dist}(\mathbf{y}, \mathbf{z}) \leq \operatorname{dist}(\mathbf{x}, \mathbf{z})$. 
Definition. Mean point $\mathbf{M}_{F}(X)$ for $X$ is a vector $\mathbf{M}_{F} \in \mathbf{R}^{m}$ such that $\mathbf{M}_{F}(X)=\underset{\mathbf{y} \in R^{m}}{\arg \min _{i=1 . . N}}\left(\operatorname{dist}\left(\mathbf{y}, \mathbf{x}_{i}\right)\right)^{2}$.

In this form the definition of the mean point goes back to Fréchet (1948). Notice that in this definition the mean point by Fréchet can be non-unique. However, this definition allows multiple useful generalisations including using it in the abstract metric spaces. It is easy to show that in the case of complete data and the Euclidean distance function $\operatorname{dist}(\mathbf{x}, \mathbf{y})=\sqrt{\sum_{i=1}^{m}\left(\mathbf{x}^{i}-\mathbf{y}^{i}\right)^{2}}$, or, more generally, in the case of any quadratic distance function (for example, Mahalanobis distance), the mean point is the standard expectation $\mathbf{M}_{F}(X)=\frac{1}{N} \sum_{i=1}^{N} \mathbf{x}^{i} \equiv E(X)$.

Definition. Orthogonal projection $\mathrm{P}(\mathbf{x}, Y)$ is defined for an object $\mathbf{x}$ and a set (not necessarily finite) of vectors $Y$ as a vector in $Y$ such that $P(\mathbf{x}, Y)=\arg \min _{\mathbf{y} \in Y} \operatorname{dist}(\mathbf{x}, \mathbf{y})$. Notice that in principle, one can have non-unique and even infinitely many projections of $\mathbf{x}$ on $Y$.

Definition. Mean squared distance $\operatorname{MSD}(X, Y)$ between a dataset $X$ and a set of vectors $Y$ is defined as $\operatorname{MSD}(X, Y)=\sqrt{\frac{1}{N} \sum_{i=1}^{N} \operatorname{dist}^{2}\left(\mathbf{x}^{i}, P\left(\mathbf{x}^{i}, Y\right)\right)}$. We will also consider a simple generalisation of MSD: weighted mean squared distance $\operatorname{MSD}_{W}(X, Y)=\sqrt{\frac{1}{\sum_{i=1}^{N} w_{i}} \cdot \sum_{i=1}^{N} w_{i} \operatorname{dist}^{2}\left(\mathbf{x}^{i}, P\left(\mathbf{x}^{i}, Y\right)\right)}$, where $w_{i}>0$ is a weight for the object $\mathbf{x}_{i}$.

Our objective in the rest of the chapter is to briefly describe the methods for constructing various approximations (principal objects) for a dataset $X$. In almost all cases the principal objects will be represented as a finite or infinite set of vectors $Y \in \mathbf{R}^{m}$ such that 1$)$ it approximates the finite dataset $X$ in the sense of minimisation of $\operatorname{MSD}(X, Y)$, and 2$)$ it answers some regularity conditions that will be discussed below.

\section{Probabilistic InTERPETATION OF STATISTICS AND NOTION OF SELF-CONSISTENCY}

In his original works, Pearson followed the principle that the only reality in data analysis is the dataset, embedded in a multidimensional metric space. This approach can be called geometrical. During the $20^{\text {th }}$ century, probabilistic interpretation of statistics was actively developed. Accordingly to this interpretation, a dataset $X$ is one particular of i.i.d. sample from a multidimensional probability distribution $F(\mathbf{x})$ which defines a probability of appearance of a sample in the point $\mathbf{x} \in \mathbf{R}^{m}$. 
The probability distribution, if can be estimated, provides a very useful auxiliary object allowing to define many notions in the theory of statistical data analysis. In particular, it allows to define principal manifolds as self-consistent objects.

The notion of self-consistency was first introduced by Efron (1967) and developed in the works of Flury (Tarpey \& Flury, 1996), where it is claimed to be one of the most fundamental in statistical theory.

Definition. Given probability distribution $F(\mathbf{x})$ and a set of vectors $Y$ we say that $Y$ is self-consistent with respect to $F(\mathbf{x})$ if for all vectors $\mathbf{y} \in Y$, one has $\mathbf{y}=M_{F}\left(\left.F\right|_{\mathbf{y}}\right)$ where $\left.F\right|_{\mathbf{y}}=\{\mathbf{x}: \mathbf{y}=P(\mathbf{x}, Y)\}$. In words, it means that any vector $\mathbf{y} \in Y$ is a conditional mean point for all points from the support of $F(\mathbf{x})$ that are orthogonally projected in $\mathbf{y}$.

The disadvantage of this definition for finite datasets is that it is not always possible to calculate the conditional mean, since in some points $\mathbf{y} \in Y$ it can be that zero or only one point is projected from $X$. This means that for finite datasets we should develop coarse-grained self-consistency notion. Usually it means that for every point $\mathbf{y} \in Y$ one defines some kind of neighbourhood and introduces a modified self-consistency with respect to this neighbourhood instead of $\mathbf{y}$ itself. Concrete implementations of this idea are described further in this chapter. In all cases, the effective size of the neighbourhood is a fundamental parameter in controlling the complexity of the resulting approximator $Y$.

\section{FOUR APPROACHES TO CLASSICAL PCA}

We can define linear principal manifolds as mean squared distance data approximators, constructed from linear manifolds embedded in $\mathbf{R}^{m}$. In fact, this corresponds to the original definition of principal lines and planes by Pearson (Pearson, 1901). However, PCA method was re-invented in other fields and even obtained different names (Karhunen-Loève or KL decomposition (Karhunen, 1946; Loève, 1955), Hotteling transform (Hotelling, 1933), Proper Orthogonal Decomposition (Lumley, 1967)) and others. Here we formulate four equivalent ways to define principal components that the user can meet in different applications.

Let us consider a linear manifold $L_{k}$ of dimension $k$ in the parametric form $L_{k}=\left\{\mathbf{a}_{0}+\beta_{1} \mathbf{a}_{1}+\ldots+\beta_{\mathrm{k}} \mathbf{a}_{\mathrm{k}} \mid \beta_{i} \in \mathbf{R}\right\}, \mathbf{a}_{0} \in \mathbf{R}^{m}$ and $\left\{\mathbf{a}_{1}, \ldots, \mathbf{a}_{\mathrm{k}}\right\}$ is a set of orthonormal vectors in $\mathbf{R}^{m}$.

Definition of PCA problem \#1 (data approximation by lines and planes):

PCA problem consists in finding such sequence $L_{k}(k=1,2, \ldots, m-1)$ that the sum of squared distances from data points to their orthogonal projections on $L_{k}$ is minimal over all linear manifolds of dimension $k$ embedded in $\mathbf{R}^{m}$ : $\operatorname{MSD}\left(X, L_{k}\right) \rightarrow \min (k=1,2, \ldots, m-1)$.

Definition of PCA problem \#2 (variance maximisation):

For a set of vectors $X$ and for a given $\mathbf{a}_{i}$, let us construct a one-dimensional distribution $\mathrm{B}^{i}=\left\{\beta: \beta=\left(\mathbf{x}, \mathbf{a}_{i}\right), \mathbf{x} \in X\right\}$ where $(\cdot ;)$ denotes scalar vector product. 
Then let us define empirical variance of $X$ along $\boldsymbol{a}_{i}$ as $\operatorname{Var}\left(\mathrm{B}^{i}\right)$, where $\operatorname{Var}()$ is the standard empirical variance. PCA problem consists in finding such $L_{k}$ that the sum of empirical dispersions of $X$ along $\mathbf{a}_{1}, \ldots, \mathbf{a}_{\mathrm{k}}$ would be maximal over all linear manifolds of dimension $k$ embedded in $\mathbf{R}^{m}: \sum_{i=1 . . k} \operatorname{Var}\left(\mathrm{B}^{i}\right) \rightarrow \max$. Let us also consider an orthogonal complement $\left\{\mathbf{a}_{k+1}, \ldots, \mathbf{a}_{\mathrm{m}}\right\}$ of the basis $\left\{\mathbf{a}_{1}, \ldots, \mathbf{a}_{\mathrm{k}}\right\}$. Then an equivalent definition (minimization of residue dispersion) is $\sum_{i=k+1}^{m} \operatorname{Var}\left(\mathrm{B}^{i}\right) \rightarrow \min$

Definition of PCA problem \#3 (mean point-to-point squared distance maximisation):

PCA problem consists in finding such sequence $L_{k}$ that the mean point-to-point squared distance between the orthogonal projections of data points on $L_{k}$ is maximal over all linear manifolds of dimension $k$ embedded in $\mathbf{R}^{m}$ : $\frac{1}{N} \sum_{i, j=1}^{N} \operatorname{dist}^{2}\left(P\left(\mathbf{x}^{i}, L_{k}\right), P\left(\mathbf{x}^{j}, L_{k}\right)\right) \rightarrow \max$. Having in mind that all linear mappings into lower-dimensional space lead to contraction of all point-to-point distances (except for some that do not change), this is equivalent to minimisation of mean squared distance distortion: $\sum_{i, j=1}^{N}\left[\operatorname{dist}^{2}\left(\mathbf{x}^{i}, \mathbf{x}^{j}\right)-\operatorname{dist}^{2}\left(P\left(\mathbf{x}^{i}, L_{k}\right), P\left(\mathbf{x}^{j}, L_{k}\right)\right)\right] \rightarrow \min$.

In the three above mentioned definitions, the basis vectors are defined up to an arbitrary rotation that does not change the manifold. To make the choice unique, in the PCA method the following principle is applied: given $\left\{\boldsymbol{a}_{0}, \boldsymbol{a}_{1}, \ldots, \boldsymbol{a}_{\mathrm{k}}\right\}$, any 'embedded' linear manifold of smaller dimension $s$ in the form $L_{s}=\left\{\boldsymbol{a}_{0}+\beta_{1} \boldsymbol{a}_{1}+\ldots+\beta_{s} \boldsymbol{a}_{s} \mid \beta_{i} \in \mathbf{R}, s<k\right\}$, must be itself a linear principal manifold of dimension $s$ for $X$.

Definition of PCA problem \#4 (correlation cancellation):

Find such an orthonormal basis $\left(\mathbf{a}_{1}, \ldots, \mathbf{a}_{\mathrm{s}}\right)$ in which the covariance matrix for $\mathbf{x}$ is diagonal. Evidently, in this basis the distributions $\left(\mathbf{a}_{i}, \mathbf{x}\right)$ and $\left(\mathbf{a}_{j}, \mathbf{x}\right)$, for $i \neq j$, have zero correlation.

Definitions 1-3 were given for finite datasets while definition 4 is sensible both for finite datasets and random vector $\mathbf{x}$. For finite datasets the empiric correlation should be cancelled. The empiric principal components which annul empiric correlations could be considered as an approximation to the principal components of the random vector.

Equivalence of above-mentioned definitions in the case of complete data and Euclidean space follows from Pythagorean theorem and elementary algebra. However, in practice this or that definition can be more useful for computations or generalisations of the PCA approach. Thus, only definitions \#1 and \#3 are suitable for working with incomplete data since they are defined with use of only distance function that can be easily calculated for the 'gapped' data vectors (see further). The definition \#1 can be generalized by weighting data points (Cochran \& Horne, 1977), while the definition \#3 can be generalized by weighting pairs of data points (Gabriel \& Zamir, 1979). More details about PCA and generalisations could be found in the fundamental book by Jollliffe (2002). 
BASIC EXPECTATION/MAXIMISATION ITERATIVE ALGORITHM FOR FINDING PRINCIPAL OBJECTS

Most of the algorithms for finding principal objects for a given dataset $X$ are constructed accordingly to the classical expectation/maximisation (EM) splitting scheme that was first formulated as a generic method by Dempster et al (1977):

Generic Expectation-Maximisation algorithm for estimating principal objects

1) Initialisation step. Some initial configuration of the principal object $Y$ is generated;

2) Expectation (projection) step. Given configuration of $Y$, calculate orthogonal projections $P(\mathbf{x}, Y)$, for all $\mathbf{x} \in X$;

3) Maximisation step. Given the calculated projections, find more optimal configuration of $Y$ with respect to $X$.

4) (Optional) adaptation step. Using some strategy, change the properties of $Y$ (typically, add or remove points to $Y$ ).

5) Repeat steps 2-4 until some convergence criteria would be satisfied.

For example, for the principal line, we have the following implementation of the above mentioned bi-iteration scheme (Bauer, 1957; for generalisations see works of Roweis (1998) and Gorban \& Rossiev (1999)).

$\underline{\text { Iterative algorithm for calculating the first principal component }}$

1) Set $\mathbf{a}_{0}=\mathbf{M}_{F}(X)$ (i.e., zero order principal component is the mean point of $X$ );

2) Choose randomly $\mathbf{a}_{1}$;

3) Calculate $b_{i}=\frac{\left(\mathbf{x}_{i}-\mathbf{a}_{0}, \mathbf{a}_{1}\right)}{\left\|\mathbf{a}_{1}\right\|^{2}}, i=1 \ldots N$;

4) Given $b_{i}$, find new $\mathbf{a}_{1}$, such that $\sum_{i=1}^{N}\left(\mathbf{x}_{i}-\mathbf{a}_{0}-\mathbf{a}_{1} b_{i}\right)^{2} \underset{\mathbf{a}_{1}}{\rightarrow} \min$, i.e. $\mathbf{a}_{1}=\frac{\sum_{i=1 . . N} \mathbf{x}_{i} b_{i}-\mathbf{a}_{0} \sum_{i=1 . . N} b_{i}}{\sum_{i=1 . . N} b_{i}^{2}} ;$

5) Re-normalize $\mathbf{a}_{1}:=\mathbf{a}_{1} /\left\|\mathbf{a}_{1}\right\|$.

6) Repeat steps 3-5 until the direction of $\mathbf{a}_{1}$ do not change more than on some small angle $\varepsilon$.

Remark. To calculate all other principal components, deflation approach is applied: after finding $\mathbf{a}_{1}$, one calculates new $X^{(1)}=X-\mathbf{a}_{0}-\mathbf{a}_{1}\left(\mathbf{x}, \mathbf{a}_{1}\right)$, and the procedure is repeated for $X^{(1)}$.

Remark. The basic EM procedure has good convergence properties only if the first eigenvalues of the empirical covariance matrix $X^{T} X$ are sufficiently well separated. If this is not the case, more sophisticated approaches are needed (Bau \& Trefethen, 1997). 
The PCA method can be treated as spectral decomposition of the symmetric and positively defined empirical covariance data matrix (defined in the case of complete data) $C=\frac{1}{N-1} X^{T} X \quad$ or $C_{i j}=\frac{1}{N-1} \sum_{k=1}^{N} x_{i}^{k} x_{j}^{k}$, where without loss of generality we suppose that the data are centered.

Definition. We call $\sigma>0$ a singular value for the data matrix $X$ iff there exist two vectors of unit length $\mathbf{a}_{\sigma}$ and $\mathbf{b}_{\sigma}$ such that $X \mathbf{a}_{\sigma}=\sigma \mathbf{b}_{\sigma}^{T}$ and $\mathbf{b}_{\sigma} X=\sigma \mathbf{a}_{\sigma}^{T}$. Then the vectors $\mathbf{a}_{\sigma}=\left\{a_{1}^{(\sigma)}, \cdots, a_{m}^{(\sigma)}\right\}$ and $\mathbf{b}_{\sigma}=\left\{b_{1}^{(\sigma)}, \cdots, b_{N}^{(\sigma)}\right\}$ are called left and right singular vectors for the singular value $\sigma$.

If we know all $p$ singular values of $X$, where $p=\operatorname{rank}(X) \leq \min (N, m)$, then we can represent $X$ as $X=\sum_{l=1}^{p} \sigma_{l} \mathbf{b}_{(l)} \mathbf{a}_{(l)}$ or $x_{i}^{k}=\sum_{l=1}^{p} \sigma_{l} b_{k}^{(l)} a_{i}^{(l)}$. It is called the singular value decomposition (SVD) of $X$. It is easy to check that the vectors $\mathbf{a}_{(l)}$ correspond to the principal vectors of $X$ and the eigenvectors of the empirical covariance matrix $C$, whereas $\mathbf{b}_{(l)}$ contain projections of $N$ points onto the corresponding principal vector. Eigenvalues $\lambda_{l}$ of $C$ and singular values $\sigma_{l}$ of $X$ and are connected by $\lambda_{l}=\frac{1}{N-1}\left(\sigma_{l}\right)^{2}$.

The mathematical basis for SVD was introduced by Sylvester (1889) and it represents a solid mathematical foundation for PCA (Strang, 1993). Although formally the problems of spectral decomposition of $X$ and eigen decomposition of $C$ are equivalent, the algorithms for performing singular decomposition directly (without explicit calculation of $C$ ) can be more efficient and robust (Bau III \& Trefethen, 1997). Thus, the iterative EM algorithm for calculating the first principal component described in the previous chapter indeed performs singular decomposition (for centered data we simply put $\mathbf{a}_{0}=\mathbf{0}$ ) and find right singular (principal) and left singular vectors one by one.

\section{K-MEANS AND PRINCIPAL POINTS}

$k$-means clustering goes back to 1950s (Steinhaus (1956); Lloyd (1957); and MacQueen (1967)). It is another extreme in its simplicity case of finding a principal object. In this case it is simply unstructured finite (and usually, much smaller than the number of points $N$ in the dataset $X$ ) set of vectors (centroids). One can say that the solution searched by $k$-means algorithm is a set of $k$ principal points (Flury, 1990).

Definition. A set of $k$ points $Y=\left\{\mathbf{y}_{1}, . ., \mathbf{y}_{k}\right\}, \mathbf{y}_{i} \in \mathbf{R}^{m}$ is called a set of principal points for dataset $X$ if it approximates $X$ with minimal mean squared distance error over all sets of $k$-points in $\mathbf{R}^{m}$ (distortion): $\sum_{\mathbf{x} \in X} \operatorname{dist}^{2}(\mathbf{x}, P(\mathbf{x}, Y)) \rightarrow \min$, where $P(\mathbf{x}, Y)$ is the point from $Y$ closest to $\mathbf{x}$. Note that the set of principal points can be not unique. 
The simplest implementation of the $k$-means procedure follows the classical EM scheme:

\section{Basic $k$-means algorithm}

1) Choose initial position of $\mathbf{y}_{1}, . ., \mathbf{y}_{k}$ randomly from $\mathbf{x}_{i} \in X$ (with equal probabilities);

2) Partition $X$ into subsets $K_{i}, i=1 . . k$ of data points by their proximity to $\mathbf{y}_{\mathrm{k}}$ : $K_{i}=\left\{\mathbf{x}: \mathbf{y}_{i}=\arg \min _{\mathbf{y}_{i} \in Y} \operatorname{dist}\left(\mathbf{x}, \mathbf{y}_{j}\right)\right\}$;

3) Re-estimate $\mathbf{y}_{i}=\sum_{\mathbf{x} \in K_{i}} \mathbf{x}, i=1 . . k$;

4) Repeat steps 2-3 until complete convergence.

The method is sensitive to the initial choice of $\mathbf{y}_{1}, . ., \mathbf{y}_{k}$. Arthur \& Vassilvitskii (2007) demonstrated that the special construction of probabilities instead of equidistribution gives serious advantages. The first centre, $\mathbf{y}_{1}$, they select equiprobable from $X$. Let the centres $\mathbf{y}_{1}, . ., \mathbf{y}_{j}$ are chosen $(j<k)$ and $D(\mathbf{x})$ be the squared shortest distance from a data point $\mathbf{x}$ to the closest centre we have already chosen. Then, we select the next centre, $\mathbf{y}_{j+1}$, from $\mathbf{x}_{i} \in X$ with probability $p\left(\mathbf{x}_{i}\right)=D\left(\mathbf{x}_{i}\right) / \sum_{\mathbf{x} \in X} D(\mathbf{x})$.

Evidently, any solution of $k$-means procedure converges to a self-consistent set of points $Y=\left\{\mathbf{y}_{1}, . ., \mathbf{y}_{k}\right\}$ ( because $Y=E[P(X, Y)]$ ), but this solution may give a local minimum of distortion and is not necessary the set of principal points (which is the globally optimal approximator from all possible $k$-means solutions).

Multiple generalisations of $k$-means scheme have been developed (see, for example, a book of Mirkin (2005) based on the idea of "data recovering"). The most computationally expensive step of the algorithm, partitioning the dataset by proximity to the centroids, can be significantly accelerated using $k d$-tree data structure (Pelleg \& Moore, 1999). Analysis of the effectiveness of EM algorithm for the $k$-means problem was given by Ostrovsky et al. (2006).

Notice that the case of principal points is the only in this chapter when selfconsistency and coarse-grained self-consistency coincide: centroid $\mathbf{y}_{k}$ has zerowidth neighbourhood and it is the conditional mean point for the data points belonging to the Voronoi region associated with $\mathbf{y}_{k}$.

\section{LOCAL PCA}

The term 'Local PCA' was first used by Braverman (1970) and Fukunaga \& Olsen (1971) to denote the simplest cluster-wise PCA approach which consists in 1) applying $k$-means or other type of clustering to a dataset and 2) calculating the principal components for each cluster separately. However, this simple idea performs rather poorly in applications, and more interesting approach consists in generalizing $k$-means by introducing principal hyperplane segments proposed by Diday (1979) and called ' $k$-segments' or local subspace analysis in a more advanced version (Liu, 2003). The algorithm for their estimation follows the classical EM scheme. 
Further development of the local PCA idea went in two main directions. First, Verbeek (2002) proposed a variant of the ' $k$-segment' approach for onedimensional segments accompanied by a strategy to assemble disconnected line segments into the global piecewise linear principal curve. Einbeck et al (2008) proposed an iterative cluster splitting and joining approach (recursive local PCA) which helps to select the optimal number and configuration of disjoined segments.

Second direction is associated with a different understanding of 'locality'. It consists in calculating local mean points and local principal directions and following them starting from (may be multiple) seed points. Locality is introduced using kernel functions defining the effective radius of neighborhood in the data space. Thus, Delicado (2001) introduced principal oriented points (POP) based on the variance maximisation-based definition of PCA (\#2 in our chapter). POPs are different from the principal points introduced above because they are defined independently one from another, while the principal points are defined globally, as a set. POPs can be assembled into the principal curves of oriented points (PCOP). Einbeck (2005) proposed a simpler approach based on local tracing of principal curves by calculating local centers of mass and the local first principal components.

SOM APPROACH FOR PRINCIPAL MANIFOLD APPROXIMATION AND ITS GENERALISATIONS

Kohonen in his seminal paper (Kohonen, 1982) proposed to modify the $k$-means approach by introducing connections between centroids such that a change in the position of one centroid would also change the configuration of some neighboring centroids. Thus Self-Organizing Maps (SOM) algorithm was developed.

With the SOM algorithm (Kohonen, 1982) we take a finite metric space $V$ with metric $\rho$ and try to map it into $\mathbf{R}^{m}$ with (1) the best preservation of initial structure in the image of $V$ and (2) the best approximation of the dataset $X$. In this way, SOMs give the most popular approximations for principal manifolds: we can take for $V$ a fragment of a regular $s$-dimensional grid and consider the resulting SOM as the approximation to the $s$-dimensional principal manifold (see, for example, (Mulier \& Cherkassky, 1995; Ritter et al, 1992)).

The SOM algorithm has several setup variables to regulate the compromise between these goals. In the original formulation by Kohonen, we start from some initial approximation of the map, $\phi_{1}: V \rightarrow \mathbf{R}^{m}$. Usually this approximation lies on the $s$-dimensional linear principal manifold. On each $k$-th step of the algorithm we have a randomly chosen datapoint $\mathbf{x} \in X$ and a current approximation $\phi_{k}: V \rightarrow \mathbf{R}^{m}$. For these $\mathbf{x}$ and $\phi_{k}$ we define an "owner" of $\mathbf{x}$ in $V: v_{x}=\operatorname{argmin}_{v \in V}\left\|\mathbf{x}-\phi_{k}(v)\right\|$. The next approximation, $\phi_{k+1}$, is $\phi_{k+1}(v)=h_{k} \times w\left(\rho\left(v, v_{x}\right)\right)\left(\mathbf{x}-\phi_{k}(v)\right)$. Here $h_{k}$ is a step size, $0 \leq w\left(\rho\left(v, v_{x}\right)\right) \leq 1$ is a monotonically decreasing neighborhood function. This process proceeds in several epochs, with neighborhood radius decreasing during each next epoch. 
The idea of SOM is flexible, was applied in many domains of science, and it lead to multiple generalizations (see the review paper by Yin (2008)). Some of the algorithms for constructing SOMs are of EM type described above, such as the Batch SOM Algorithm (Kohonen, 1997): it includes projecting step exactly the same as in $k$-means, and the maximization step at which all $\phi(v)$ are modified simultaneously.

One source of theoretical insatisfaction with SOM is that it is not possible to define an optimality criterion (Erwin et al, 1992): SOM is a result of the algorithm at work and there does not exist any objective function that is minimized by the training process.

In attempt to resolve this issue, Bishop et al. (1998) developed the optimizationbased Generative Topographic Mapping (GTM) method. In this setting, it is supposed that the observed data is i.i.d. sample from a mixture of Gaussian distributions with the centers aligned along a two-dimensional grid, embedded in the data space. Parameters of this mixture are determined by EM-based maximization of the likelihood function (probability of observing $X$ within this data model).

\section{Principal ManifoldS by Hastie AND StUelze}

Principal curves and principal two dimensional surfaces for a probability distribution $F(\mathbf{x})$ were introduced in the $\mathrm{PhD}$ thesis by Trevor Hastie (1984) as a self-consistent (non-linear) one- and two-dimensional globally parametrisable smooth manifolds without self-intersections.

Definition. Let $G$ be the class of differentiable 1-dimensional curves in $\mathbf{R}^{m}$, parametrized by $\lambda \in \mathbf{R}^{1}$ and without self-intersections. The Principal Curve of the probability distribution $F(\mathbf{x})$ is such a $Y(\lambda) \in G$ that is self-consistent.

Remark. Usually, a compact subset of $\mathbf{R}^{m}$ and a compact interval of parameters $\lambda \in \mathbf{R}^{1}$ are considered. To discuss unbounded regions, it is necessary to add a condition that $Y(\lambda)$ has finite length inside any bounded subset of $\mathbf{R}^{m}$ (Kégl, 1999).

Definition. Let $G^{2}$ be the class of differentiable 2-dimensional surfaces in $\mathbf{R}^{m}$, parametrized by $\lambda \in \mathbf{R}^{2}$ and without self-intersections. The Principal Surface of the probability distribution $F(\mathbf{x})$ is such a $Y(\lambda) \in G^{2}$ that is self-consistent. (Again, for unbounded regions it is necessary to assume that for any bounded $\operatorname{set} B$ from $\mathbf{R}^{m}$ the set of parameters $\lambda$ for which $Y(\lambda) \in B$ is also bounded.)

First, Hastie and Stuelze proposed an algorithm for finding the principal curves and principal surfaces for a probability distribution $F(\mathbf{x})$, using the classical EM splitting. We do not provide this algorithm here because for a finite dataset $X$ it can not be directly applied because in a typical point on $Y(\lambda)$ only zero or one data point is projected, hence, one can not calculate the expectation. As mentioned above, in this case we should use some kind of coarse-grained self-consistency. In the original approach by Hastie (1984), this is done through introducing 
smoothers. This gives the practical formulation of the HS algorithm for estimating the principal manifolds from a finite dataset $X$ :

$\underline{\text { Hastie and Stuelze algorithm for finding principal curve for finite dataset }}$

1) Initialize $Y(\lambda)=\mathbf{a}_{0}+\lambda \mathbf{a}_{1}$, where $\mathbf{a}_{0}$ is a mean point and $\mathbf{a}_{1}$ is the first principal component;

2) Project every data point $\mathbf{x}_{i}$ onto $Y(\lambda)$ : i.e., for each $\mathbf{x}_{i}$ find $\lambda_{i}$ such a that $Y\left(\lambda_{i}\right)=\underset{\lambda}{\operatorname{arginf}}\left\|Y(\lambda)-\mathbf{x}_{i}\right\|^{2}$. In practice it requires interpolation procedure because $Y(\lambda)$ is determined in a finite number of points $\left\{\lambda_{1}, \ldots, \lambda_{N}\right\}$. The simplest is the piecewise interpolation procedure, but more sophisticated procedures can be proposed (Hastie, 1984);

3) Calculate new $Y^{\prime}(\lambda)$ in the finite number of internal coordinates $\left\{\lambda_{1}, \ldots, \lambda_{N}\right\}$ (found at the previous step) as the local average of points $\mathbf{x}_{i}$ and some other points, that have close to $\lambda_{i}$ projections onto $Y$. To do this, 1) a span $[w \times N]$ is defined ([.] here is integer part), where $0<w<<1$ is a parameter of the method (coarse-grained self-consistency neighbourhood radius); 2) for $[w \times N]$ internal coordinates $\left\{\lambda_{i_{1}}, \ldots, \lambda_{i_{[w \times N]}}\right\}$ closest to $\lambda_{i}$ and the corresponding $\left\{\mathbf{x}_{i_{1}}, \ldots, \mathbf{x}_{i_{[\mathrm{w} \times \mathrm{N}}}\right\}$ calculate weighted least squares linear regression $\left.y(\lambda)=\mathbf{a}^{(i)} \lambda+\mathbf{b}^{(i)} ; 3\right)$ define $Y^{\prime}\left(\lambda_{i}\right)$ as the value of the linear regression in $\lambda_{i}: Y^{\prime}\left(\lambda_{i}\right)=\mathbf{a}^{(i)} \lambda_{l}+\mathbf{b}^{(i)}$.

4) Reassign $Y(\lambda) \leftarrow Y^{\prime}(\lambda)$

5) Repeat steps 2)-4) until $Y$ does not change (approximately).

Remark. For the weights in the regression at the step 3) Hastie proposed to use some symmetric kernel function that vanishes on the borders of the neighbourhood. For example, for $\mathbf{x}_{i}$ let us denote as $\lambda_{i_{[\mathrm{w} \times \mathrm{N}]}}$ the most distant value of the internal coordinate from $[w \times N]$ ones closest to $\lambda_{i}$. Then we can define weight for the pair $\left(\lambda_{i_{\mathrm{j}}}, \mathbf{x}_{i_{\mathrm{j}}}\right)$ as

$$
\omega_{j}^{i}=\left\{\begin{array}{c}
\left(1-\left(\left|\lambda_{i_{j}}-\lambda_{i}\right| /\left|\lambda_{i_{j}}-\lambda_{i_{N}}\right|\right)^{3}\right)^{1 / 3}, \text { if }\left|\lambda_{i_{j}}-\lambda_{i}\right|<\left|\lambda_{i_{j}}-\lambda_{i_{N}}\right|, \\
0, \text { otherwise. }
\end{array}\right.
$$

Remark. At the step 3) an alternative approach was also proposed with use of cubic splines to approximate the smooth function $Y^{\prime}(\lambda)$ from all pairs $\left(\lambda_{i}, \mathbf{x}_{i}\right), i=1 . . N$.

Non-linear Principal Manifolds constructed by this algorithm are usually called Hastie-Stuelze (HS) principal manifolds. However, the global optimality of HS principal manifolds is not guaranteed (only self-consistency in the case of distribution or coarse-grained self-consistency in the case of dataset is guaranteed by construction). For example, the second principal component of a sample $X$ from a normal distribution is self-consistent and will be correct HS principal curve but of course not the most optimal.

We should also underline that our view on what is the object constructed by the HS algorithm for a dataset $X$ depends on 1) probabilistic interpretation of the nature of $X$, and 2) the chosen heuristic approach to coarse-grained selfconsistency. If we do not suppose that the dataset is generated by i.i.d. sampling 
from $F(\mathbf{x})$ then the definition of HS principal manifold is purely operational: HS principal manifold for $X$ is the result of application of HS algorithm for finite datasets. Analogous remark is applicable for all principal manifold approximators constructed for finite datasets and described further in this chapter.

In his $\mathrm{PhD}$ thesis Hastie noticed that the HS principal curve does not coincide with the generating curve in a very simple additive data generation model

$$
X=f(\lambda)+\varepsilon,
$$

where $f(\lambda)$ is some curve embedded in data space and $\varepsilon$ is noise distribution independent on $\lambda$. Because of the fact that if $f(\lambda)$ is not a straight line then it is not self-consistent, HS principal curves were claimed to be "biased". This inspired Tibshirani (1992) to introduce an alternative definition of the principal curve, based directly on a continuous mixture model (1) and maximising regularized likelihood.

\section{KÉGL-KRYZHAK IMPROVEMENT}

Kégl in his PhD thesis supervised by Kryzhak (Kégl, 1999) revised the existing methods for estimating the principal curves. In particular, this led to the definition of principal curves with limited length.

Definition. Principal curve $Y_{L}(\lambda)$ of length $L$ is such a curve that the mean squared distance from the dataset $X$ to the curve $Y_{L}(\lambda)$ is minimal over all curves of length less than or equal to $L: \sum_{i=1}^{N} \operatorname{dist}^{2}\left(\mathbf{x}^{i}, P\left(\mathbf{x}^{i}, Y_{L}\right)\right) \rightarrow$ min.

Theorem. Assume that $X$ has finite second moments, i.e. $\sum_{i=1}^{N} \mathbf{x}^{i}\left(\mathbf{x}^{i}\right)^{T}<\infty$. Then for any $L>0$ there exists a principal curve of length $L$.

Principal curves of length $L$ as defined by Kégl, are globally optimal approximators as opposite to the HS principal curves that are only self-consistent. However, all attempts to construct a practical algorithm for finding globally optimal principal curves of length $L$ were not successful. Instead Kégl developed an efficient heuristic Polygonal line algorithm for constructing piecewise linear principal curves.

Let us consider a piecewise curve $Y$ composed from vertices located in points $\left\{\mathbf{y}^{1}, \ldots, \mathbf{y}^{k+1}\right\}$ and $k$ segments connecting pairs of vertices $\left\{\mathbf{y}^{j}, \mathbf{y}^{j+1}\right\}, j=1 . . k$. Kégl's algorithm searches for a (local) optimum of the penalised mean squared distance error function:

$$
U(X, Y)=\operatorname{MSD}(X, Y)+\frac{\lambda}{k+1} \sum_{i=1}^{k+1} \mathrm{CP}(i),
$$

where $\mathrm{CP}(i)$ is a curvature penalty function for a vertex $i$ chosen as 


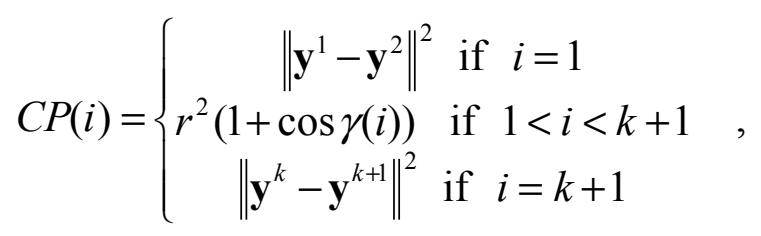

where $\cos \gamma(i)=\frac{\left(\mathbf{y}^{i-1}-\mathbf{y}^{i}, \mathbf{y}^{i+1}-\mathbf{y}^{i}\right)}{\left\|\mathbf{y}^{i-1}-\mathbf{y}^{i}\left|\left\|\mid \mathbf{y}^{i+1}-\mathbf{y}^{i}\right\|\right.\right.}$ is the cosinus of the angle between two neighbouring segments at the vertex $i, r=\operatorname{maxdist}_{\mathbf{x} \in X}\left(\mathbf{x}, \mathbf{M}_{\mathbf{F}}(X)\right)$ is the 'radius' of the dataset $X$, and $\lambda$ is a parameter controlling the curve global smoothness.

The Polygonal line algorithm (Kégl, 1999) follows the standard EM splitting scheme:

$\underline{\text { Polygonal line algorithm for estimating piece-wise linear principal curve }}$

1) The initial approximation is constructed as a segment of principal line. The length of the segment is the difference between the maximal and the minimal projection value of $X$ onto the first principal component. The segment is positioned such that it contains all of the projected data points. Thus in the initial approximation one has two vertices $\left\{\mathbf{y}^{1}, \mathbf{y}^{2}\right\}$ and one segment between them $(k=1)$.

2) Projection step. The dataset $X$ is partitioned into $2 k+1$ $K_{z}=\left\{\mathbf{x}: z=\arg \min _{z \in \text { verticesnsegments }} \operatorname{dist}(\mathbf{x}, z)\right\} \quad$ subsets constructed by their proximity to $k+1$ vertices and $k$ segments. If a segment $i$ and a vertex $j$ are equally distant from $\mathbf{x}$ then $\mathbf{x}$ is placed into $K_{j}$ only.

3) Optimisation step. Given partitioning obtained at the step 2, the functional $U(X, Y)$ is optimised by use of a gradient technique. Fixing partitioning into $K_{i}$ is needed to calculate the gradient of $U(X, Y)$ because otherwise it is not a differentiable function with respect to the position of vertices $\left\{\mathbf{y}_{i}\right\}$.

4) Adaptation step. Choose the segment with the largest number of points projected onto it. If more than one such segment exists then the longest one is chosen. The new vertex is inserted in the midpoint of this segment; all other segments are renumerated accordingly.

5) Stopping criterion. The algorithm stops when the number of segments exceeds $\beta \cdot N^{1 / 3} \cdot \frac{r}{\operatorname{MSD}(X, Y)}$.

Heuristically, the default parameters of the method have been proposed $\beta=0.3$, $\lambda=\lambda^{\prime} \cdot \frac{k}{N^{1 / 3}} \cdot \frac{\operatorname{MSD}(X, Y)}{r}, \lambda^{\prime}=0.13$. The details of implementation together with convergence and computational complexity study are provided elsewhere (Kégl, 1999).

Smola et al. (2001) proposed a regularized principal manifolds framework, based on minimization of quantization error functional with a large class of regularizers that can be used and a universal EM-type algorithm. For this algorithm, the convergence rates were analyzed and it was showed that for some regularizing 
terms the convergence can be optimized with respect to the Kegl's polygonal line algorithm.

\section{ELASTIC MAPS APPROACH}

In a series of works (Gorban \& Rossiev, 1999; Gorban et al., 2001, 2003; Gorban \& Zinovyev, 2005; Gorban et al., 2007, 2008), the authors of this chapter used metaphor of elastic membrane and plate to construct one-, two- and threedimensional principal manifold approximations of various topologies. Mean squared distance approximation error combined with the elastic energy of the membrane serves as a functional to be optimised. The elastic map algorithm is extremely fast at the optimisation step due to the simplest form of the smoothness penalty. It is implemented in several programming languages as software libraries or front-end user graphical interfaces freely available from the web-site http://bioinfo.curie.fr/projects/vidaexpert. The software found applications in microarray data analysis, visualization of genetic texts, visualization of economical and sociological data and other fields (Gorban et al, 2001, 2003; Gorban \& Zinovyev 2005; Gorban et al, 2007, 2008).

Let $G$ be a simple undirected graph with set of vertices $V$ and set of edges $E$.

Definition. $k$-star in a graph $G$ is a subgraph with $k+1$ vertices $v_{0,1, \ldots, k} \in V$ and $k$ edges $\left\{\left(v_{0}, v_{i}\right) \mid i=1, . ., k\right\} \in E$. The $r i b$ is by definition a 2 -star.

Definition. Suppose that for each $k \geq 2$, a family $S_{k}$ of $k$-stars in $G$ has been selected. Then we define an elastic graph as a graph with selected families of $k$-stars $S_{k}$ and for which for all $E^{(i)} \in E$ and $S_{k}^{(j)} \in S_{k}$, the corresponding elasticity moduli $\lambda_{i}>0$ and $\mu_{k j}>0$ are defined.

Definition. Suppose that for each $k \geq 2$, a family $S_{k}$ of $k$-stars in $G$ has been selected. Then we define an elastic graph as a graph with selected families of $k$-stars $S_{k}$ and for which for all $E^{(i)} \in E$ and $S_{k}^{(j)} \in S_{k}$, the corresponding elasticity moduli $\lambda_{i}>0$ and $\mu_{k j}>0$ are defined.

Definition. Primitive elastic graph is an elastic graph in which every non-terminal node (with the number of neighbours more than one) is associated with a $k$-star formed by all neighbours of the node. All $k$-stars in the primitive elastic graph are selected, i.e. the $S_{k}$ sets are completely determined by the graph structure.

Definition. Let $E^{(i)}(0), E^{(i)}(1)$ denote two vertices of the graph edge $E^{(i)}$ and $S_{k}^{(j)}(0), \ldots, S_{k}^{(j)}(k)$ denote vertices of a $k$-star $S_{k}^{(j)}$ (where $S_{k}^{(j)}(0)$ is the central vertex, to which all other vertices are connected). Let us consider a map $\phi: V \rightarrow \mathbf{R}^{m}$ which describes an embedding of the graph into a multidimensional space. The elastic energy of the graph embedding in the Euclidean space is defined as

$$
\begin{gathered}
U^{\phi}(G):=U_{E}^{\phi}(G)+U_{R}^{\phi}(G), \\
U_{E}^{\phi}(G):=\sum_{E^{(i)}} \lambda_{i}\left\|\phi\left(E^{(i)}(0)\right)-\phi\left(E^{(i)}(1)\right)\right\|^{2},
\end{gathered}
$$




$$
U_{E}^{\phi}(G):=\sum_{S_{k}^{(j)}} \mu_{k j}\left\|\phi\left(S_{k}^{(j)}(0)\right)-\frac{1}{k} \sum_{i=1}^{k} \phi\left(S_{k}^{(j)}(i)\right)\right\|^{2} .
$$

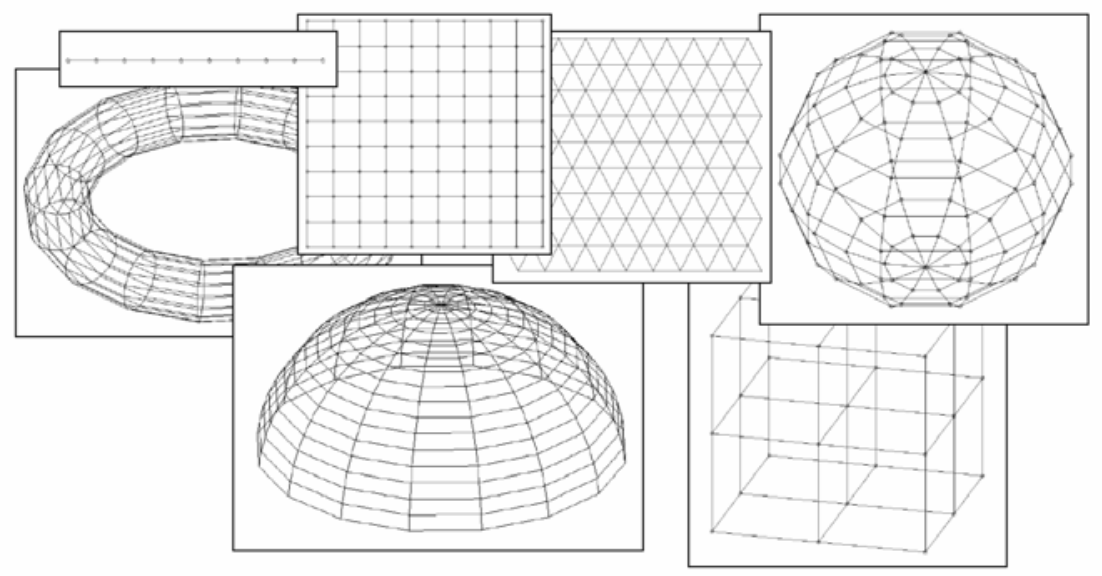

Fig. 1. Elastic nets used in practice.

Definition. Elastic net is a particular case of elastic graph which 1) contains only ribs (2-stars) in the set of the selected $k$-stars; and 2) the vertices of this graph form a regular small-dimensional grid (Fig.1). The elastic net is characterised by internal dimension $\operatorname{dim}(G)$. Every node $v_{i}$ in the elastic net is indexed by the discrete values of internal coordinates $\left\{\lambda_{1}^{i}, \ldots, \lambda_{\operatorname{dim}(G)}^{i}\right\}$ in such a way that the nodes close on the graph have similar internal coordinates. The purpose of the elastic net is to introduce point approximations to manifolds. Historically it was first explored and used in applications. To avoid confusion, one should notice that the term elastic net was independently introduced by several groups: for solving the traveling salesman problem (Durbin \&Willshaw, 1987), in the context of principal manifolds (Gorban et al, 2001) and recently in the context of regularized regression problem (Zhou \& Hastie, 2005). These three notions are completely independent and denote different things.

Definition. Elastic map is a continuous manifold $Y \in \mathbf{R}^{m}$ constructed from the elastic net as its grid approximation using some between-node interpolation procedure. This interpolation procedure constructs a continuous mapping $\phi_{c}:\left\{\lambda_{1}, \ldots, \lambda_{\operatorname{dim}(G)}\right\} \rightarrow \mathbf{R}^{m}$ from the discrete map $\phi: V \rightarrow \mathbf{R}^{m}$, used to embed the graph in $\mathbf{R}^{m}$, and the discrete values of node indices $\left\{\lambda_{1}^{i}, \ldots, \lambda_{\operatorname{dim}(G)}^{i}\right\}, i=1 \ldots|V|$. For example, the simplest piecewise linear elastic map is build by piecewise linear $\operatorname{map} \phi_{c}$.

Definition. Elastic principal manifold of dimension $s$ for a dataset $X$ is an elastic map, constructed from an elastic net $Y$ of dimension $s$ embedded in $\mathbf{R}^{m}$ using such a map $\phi_{\text {opt }}: Y \rightarrow \mathbf{R}^{m}$ that corresponds to the minimal value of the functional

$$
U^{\phi}(X, Y)=\operatorname{MSD}_{\mathrm{w}}(X, Y)+U^{\phi}(G)
$$

where the weighted mean squared distance from the dataset $X$ to the elastic net $Y$ is calculated as the distance to the finite set of vertices $\left\{\mathbf{y}^{1}=\phi\left(v_{1}\right), \ldots, \mathbf{y}^{k}=\phi\left(v_{\mathrm{k}}\right)\right\}$. 
In the Euclidean space one can apply an EM algorithm for estimating the elastic principal manifold for a finite dataset. It is based in turn on the general algorithm for estimating the locally optimal embedding map $\phi$ for an arbitrary elastic graph $G$, described below.

Optimisation of the elastic graph algorithm:

1) Choose some initial position of nodes of the elastic graph $\left\{\mathbf{y}^{1}=\phi\left(v_{1}\right), \ldots, \mathbf{y}^{k}=\phi\left(v_{\mathrm{k}}\right)\right\}$, where $k$ is the number of graph nodes $k=|V|$;

2) Calculate two matrices $e_{i j}$ and $s_{i j}$, using the following sub-algorithm:

i. Initialize the $s_{i j}$ matrix to zero;

ii. For each $k$-star $S_{k}^{(i)}$ with elasticity module $\mu_{k i}$, outer nodes $v_{N 1}, \ldots, v_{N k}$ and the central node $v_{N 0}$, the $s_{i j}$ matrix is updated as follows $(1 \leq l, m \leq k)$ :

$$
\begin{aligned}
& s_{N_{0} N_{0}} \leftarrow s_{N_{0} N_{0}}+\mu_{k i}, s_{N_{l} N_{m}} \leftarrow s_{N_{l} N_{m}}+\mu_{k i} / k^{2} \\
& s_{N_{0} N_{l}} \leftarrow s_{N_{0} N_{l}}-\mu_{k i} / k, s_{N_{l} N_{0}} \leftarrow s_{N_{l} N_{0}}-\mu_{k i} / k
\end{aligned}
$$

iii. Initialize the $e_{i j}$ matrix to zero;

iv. For each edge $E^{(i)}$ with weight $\lambda_{i}$, one vertex $v_{k 1}$ and the other vertex $v_{k 2}$, the $e_{j k}$ matrix is updated as follows:

$$
\begin{aligned}
& e_{k_{1} k_{1}} \leftarrow e_{k_{1} k_{1}}+\lambda_{i}, e_{k_{2} k_{2}} \leftarrow e_{k_{2} k_{2}}+\lambda_{i} \\
& e_{k_{1} k_{2}} \leftarrow e_{k_{1} k_{2}}-\lambda_{i}, e_{k_{2} k_{1}} \leftarrow e_{k_{2} k_{1}}-\lambda_{i}
\end{aligned}
$$

3) Partition $X$ into subsets $K_{i}, i=1 . . k$ of data points by their proximity to $\mathbf{y}_{k}: K_{i}=\left\{\mathbf{x}: \mathbf{y}_{i}=\arg \min _{\mathbf{y}_{j} \in Y} \operatorname{dist}\left(\mathbf{x}, \mathbf{y}_{j}\right)\right\}$;

4) Given $K_{i}$, calculate matrix $a_{j s}=\frac{n_{j} \delta_{j s}}{\sum_{i=1}^{N} w_{i}}+e_{j s}+s_{j s}$, where $n_{j}=\sum_{x^{i} \in K_{j}} w_{i}$, $\delta_{j s}$ is the Kronecker's symbol.

5) Find new position of $\left\{\mathbf{y}^{1}, \ldots, \mathbf{y}^{k}\right\}$ by solving the system of linear equations

$$
\sum_{s=1}^{k} a_{j s} \mathbf{y}^{s}=\frac{1}{\sum_{i=1}^{N} w_{i}} \cdot \sum_{\mathbf{x}^{i} \in K_{j}} w_{i} \mathbf{x}^{i}
$$

6) Repeat steps 3-5 until complete or approximate convergence of node positions $\left\{\mathbf{y}^{1}, \ldots, \mathbf{y}^{k}\right\}$.

As usual, the EM algorithm described above gives only locally optimal solution. One can expect that the number of local minima of the energy function $U$ grows with increasing the 'softness' of the elastic graph (decreasing $\mu_{k j}$ parameters). Because of this, in order to obtain a solution closer to the global optimum, the softening strategy has been proposed, used in the algorithm for estimating the elastic principal manifold.

\section{Algorithm for estimating the elastic principal manifold}


1) Define a decreasing set of numbers $\left\{m_{1}, \ldots, m_{p}\right\}, m_{p}=1$ (for example, $\left\{10^{3}\right.$, $\left.10^{2}, 10,1\right\}$ ), defining $p$ epochs for softening;

2) Define the base values of the elastic moduli $\lambda_{i}^{\text {(base })}$ and $\mu_{i}^{\text {(base) }}$;

3) Initialize positions of the elastic net nodes $\left\{\mathbf{y}^{1}, \ldots, \mathbf{y}^{k}\right\}$ on the linear principal manifold spanned by first $\operatorname{dim}(G)$ principal components;

4) Set epoch_counter $=1$

5) Set the the elastic moduli $\lambda_{i}=m_{\text {epoch_counter }} \lambda_{i}^{\text {(base) }}$ and $\mu_{i}=m_{\text {epoch_counter }} \mu_{i}^{\text {(base) }}$;

6) Modify the elastic net using the algorithm for optimisation of the elastic graph;

7) Repeat steps 5-6 for all values of epoch_counter $=2, \ldots, p$.

Remark. The values $\lambda_{i}$ and $\mu_{j}$ are the coefficients of stretching elasticity of every edge $E^{(i)}$ and of bending elasticity of every rib $S_{2}^{(j)}$. In the simplest case $\lambda_{1}=\lambda_{2}=\ldots=\lambda_{s}=\lambda(s), \mu_{1}=\mu_{2}=\ldots=\mu_{r}=\mu(r)$, where $s$ and $r$ are the numbers of edges and ribs correspondingly. Approximately dependence on graph 'resolution' is given by (Gorban \& Zinovyev, 2007): $\lambda(s)=\lambda_{0} \cdot s^{\frac{2-\operatorname{dim}(G)}{\operatorname{dim}(G)}}, \mu(s)=\mu_{0} \cdot r^{\frac{2-\operatorname{dim}(G)}{\operatorname{dim}(G)}}$. This formula is applicable, of course, only for the elastic nets. In general case $\lambda_{i}$, $\mu_{i}$ are often made variable in different parts of the graph accordingly to some adaptation strategy (Gorban \& Zinovyev, 2005).

Remark. $U_{E}^{\phi}(G)$ penalizes the total length (or, indirectly, "square", "volume", etc.) of the constructed manifold and provides regularization of distances between node positions at the initial steps of the softening. At the final stage of the softening $\lambda_{i}$ can be put to zero with little effect on the manifold configuration.

Elastic map post-processing such as map extrapolation can be applied to increase its usability and avoid the 'border effect', for details see (Gorban \& Zinovyev, 2008).

\section{PLURIHARMONIC GRAPHS AS IDEAL APPROXIMATORS}

Approximating datasets by one dimensional principal curves is not satisfactory in the case of datasets that can be intuitively characterized as branched. A principal object which naturally passes through the 'middle' of such a data distribution should also have branching points that are missing in the simple structure of principal curves. Introducing such branching points converts principal curves into principal graphs.

Principal graphs were introduced by Kégl \& Krzyzak (2002) as a natural extension of one-dimensional principal curves in the context of skeletonisation of hand-written symbols. The most important part of this definition is the form of the penalty imposed onto deviation of the configuration of the branching points embedment from their 'ideal' configuration. Kégl's definition of the penalty on the graph's non-linearity was problem-specific. Thus, eight vertex types were introduced: 
1) end: terminal vertices, having connectivity degree one;

2) line (rib): 2-star, the ideal configuration of incident edges forms the open angle;

3) corner: 2-star, the ideal configuration of incident edges forms the right angle;

4) fully flexible 3-star: have zero penalty;

5) $T: 3$-star, the ideal configuration forms T-shape;

6) $Y$ : 3-star, the ideal configuration is such that two neighbour vertices conincide and form the open angle with the third one;

7) fully flexible 4-star: have zero penalty;

8) $X: 4$-star, the ideal configuration is such that two crossing pairs of adjacent edges form an open angle.

Assigning types for all vertices serves for definition of the penalty on the total deviation from the graph 'ideal' configuration (Kégl, 1999). Other types of vertices were not considered, and outside the field of symbol skeletonization applicability of such a definition of principal graph remains limited.

Gorban \& Zinovyev (2005), Gorban et al. (2007) and Gorban et al. (2008) proposed to use a universal form of non-linearity penalty for the branching points. The form of this penalty is defined in the previous chapter for the elastic energy of graph embedment. It naturally generalizes the simplest three-point second derivative approximation squared:

for a 2-star (or rib) the penalty equals $\left\|\phi\left(S_{2}^{(j)}(0)\right)-\frac{1}{2}\left(\phi\left(S_{2}^{(j)}(1)\right)+\phi\left(S_{2}^{(j)}(2)\right)\right)\right\|^{2}$, for a 3-star it is $\left\|\phi\left(S_{3}^{(j)}(0)\right)-\frac{1}{3}\left(\phi\left(S_{3}^{(j)}(1)\right)+\phi\left(S_{3}^{(j)}(2)\right)+\phi\left(S_{3}^{(j)}(3)\right)\right)\right\|^{2}$, etc.

For a $k$-star this penalty equals to zero iff the position of the central node coincides with the mean point of its neighbors. An embedment $\phi(G)$ is 'ideal' if all such penalties equal to zero. For a primitive elastic graph this means that this embedment is a harmonic function on graph: its value in each non-terminal vertex is a mean of the value in the closest neighbors of this vertex.

For non-primitive graphs we can consider stars which include not all neighbors of their centers. For example, for a square lattice we create elastic graph (elastic net) using 2-stars (ribs): all vertical 2-stars and all horizontal 2-stars. For such elastic net, each non-boundary vertex belongs to two stars. For a general elastic graph $G$ with sets of $k$-stars $S_{k}$ we introduce the following notion of pluriharmoning function.

Definition. A map $\phi: V \rightarrow \mathbf{R}^{m}$ defined on vertices of $G$ is pluriharmonic iff for any $k$-star $S_{k}^{(j)} \in S_{k}$ with the central vertex $S_{k}^{(j)}(0)$ and the neighbouring vertices $S_{k}^{(j)}(i), i=1 \ldots k$, the equality holds:

$$
\phi\left(S_{k}^{(j)}(0)\right)=\frac{1}{k} \sum_{i=1}^{k} \phi\left(S_{k}^{(j)}(i)\right)
$$

Pluriharmonic maps generalize the notion of linear map and of harmonic map, simultaneously. For example: 
1) $1 \mathrm{D}$ harmonic functions are linear;

2) If we consider an $n \mathrm{D}$ cubic lattice as a primitive graph (with $2 n$-stars for all non-boundary vertices), then the correspondent pluriharmonic functions are just harmonic ones;

3) If we create from $n \mathrm{D}$ cubic lattice a standard $n \mathrm{D}$ elastic net with 2-stars (each non-boundary vertex is a center of $n$ 2-stars, one 2-stars for each coordinate direction), then pluriharmonic functions are linear.

Pluriharmonic functions have many attractive properties, for example, they satisfy the following maximum principle. A vertex $v$ of an elastic graph is called a corner point or an extreme point of $G$ iff $v$ is not a centre of any $k$-star from $S_{k}$ for all $k>0$.

Theorem. Let $\phi: V \rightarrow \mathbf{R}^{m}$ be a pluriharmonic map, $F$ be a convex function on $\mathbf{R}^{m}$, and $a=\max _{x \in V} F(\phi(x))$. Then there is a corner point $v$ of $G$ such that $F(\phi(v))=a$.

Convex functions achieve their maxima in corner points. Even particular cases of this theorem with linear functions $F$ are quite useful. Linear functions achieve their maxima and minima in corner points.

In the theory of principal curves and manifolds the penalty functions were introduced to penalise deviation from linear manifolds (straight lines or planes). We proposed to use pluriharmonic embeddings as "ideal objects" instead of manifolds and to introduce penalty (5) for deviation from this ideal form.

\section{GRAPH GRAMMARS AND THREE TYPES OF COMPLEXITY FOR PRINCIPAL GRAPHS}

The principal graphs can be called data approximators of controllable complexity. By complexity of the principal objects we mean the following three notions:

1) Geometric complexity: how far a principal object deviates from its ideal configuration; for the elastic principal graphs we explicitly measure deviation from the 'ideal' pluriharmonic graph by the elastic energy $U_{\phi}(G)$ (3) (this sort of complexity is often just a measure of non-linearity);

2) Structural complexity measure: it is some non-decreasing function of the number of vertices, edges and $k$-stars of different orders $\left.\mathrm{SC}(G)=\mathrm{SC}\left(|V|,|E|,\left|S_{2}\right|, \ldots, \mid S_{\mathrm{m}}\right)\right)$; this function penalises for number of structural elements;

3) Construction complexity is defined with respect to a graph grammar as a minimum number of applications of elementary transformations necessary to construct given $G$ from the simplest graph (one vertex, zero edges).

The construction complexity is defined with respect to a grammar of elementary transformation. The graph grammars (Löwe, 1993; Nagl, 1976) provide a welldeveloped formalism for the description of elementary transformations. An elastic graph grammar is presented as a set of production (or substitution) rules. Each rule has a form $A \rightarrow B$, where $A$ and $B$ are elastic graphs. When this rule is applied to an elastic graph, a copy of $A$ is removed from the graph together with all its incident edges and is replaced with a copy of $B$ with edges that connect $B$ to the graph. For a full description of this language we need the notion of a labeled 
graph. Labels are necessary to provide the proper connection between $B$ and the graph (Nagl, 1976). An approach based on graph grammars to constructing effective approximations of elastic principal graph was proposed recently (Gorban et al, 2007).

Let us define graph grammar $O$ as a set of graph grammar operations $O=\left\{o_{1}, \ldots, o_{\mathrm{s}}\right\}$. All possible applications of a graph grammar operation $o_{i}$ to a graph $G$ gives a set of transformations of the initial graph $o_{i}(G)=\left\{G_{1}, G_{2}, \ldots, G_{p}\right\}$, where $p$ is the number of all possible applications of $o_{i}$ to $G$. Let us also define a sequence of $r$ different graph grammars $\left\{O^{(1)}=\left\{o_{1}^{(1)}, \ldots, o_{s_{1}}^{(1)}\right\}, \cdots, O^{(r)}=\left\{o_{1}^{(r)}, \ldots, o_{s_{r}}^{(r)}\right\}\right\}$.

Let us choose a grammar of elementary transformations, predefined boundaries of structural complexity $S C_{\max }$ and construction complexity $C C_{\max }$, and elasticity coefficients $\lambda_{i}$ and $\mu_{k j}$.

Definition. Elastic principal graph for a dataset $X$ is such an elastic graph $G$ embedded in the Euclidean space by the map $\phi: V \rightarrow \mathbf{R}^{m}$ that $\operatorname{SC}(G) \leq S C_{\max }$, $\mathrm{CC}(G) \leq C C_{\max }$, and $U_{\phi}(G) \rightarrow$ min over all possible elastic graphs $G$ embeddings in $\mathbf{R}^{m}$.

\section{Algorithm for estimating the elastic principal graph}

1) Initialize the elastic graph $G$ by 2 vertices $v_{1}$ and $v_{2}$ connected by an edge. The initial map $\phi$ is chosen in such a way that $\phi\left(v_{1}\right)$ and $\phi\left(v_{2}\right)$ belong to the first principal line in such a way that all the data points are projected onto the principal line segment defined by $\phi\left(v_{1}\right), \phi\left(v_{2}\right)$;

2) For all $j=1 \ldots r$ repeat steps 3-6:

3) Apply all grammar operations from $O^{(j)}$ to $G$ in all possible ways; this gives a collection of candidate graph transformations $\left\{G_{1}, G_{2}, \ldots\right\}$;

4) Separate $\left\{G_{1}, G_{2}, \ldots\right\}$ into permissible and forbidden transformations; permissible transformation $G_{k}$ is such that $\mathrm{SC}\left(G_{k}\right) \leq \mathrm{SC}_{\max }$, where $\mathrm{SC}_{\max }$ is some predefined structural complexity ceiling;

5) Optimize the embedment $\phi$ and calculate the elastic energy $U_{\phi}(G)$ of graph embedment for every permissible candidate transformation, and choose such a graph $G_{\text {opt }}$ that gives the minimal value of the elastic functional: $G_{\text {opt }}=\arg \inf _{G_{k} \in \text { permissible set }} U_{\phi}\left(G_{k}\right)$;

6) Substitute $G \leftarrow G_{\text {opt }}$;

7) Repeat steps 2-6 until the set of permissible transformations is empty or the number of operations exceeds a predefined number - the construction complexity.

\section{PRINCIPAL TREES AND METRO MAPS}

Let us construct the simplest non-trivial type of the principal graphs, called principal trees. For this purpose let us introduce a simple 'Add a node, bisect an edge' graph grammar (see Fig. 2) applied for the class of primitive elastic graphs. 


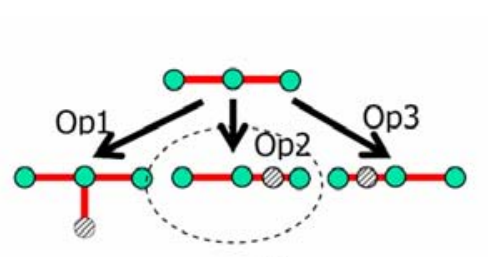

a)

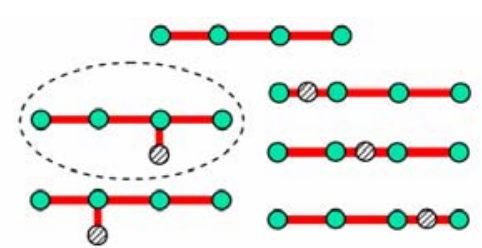

b)

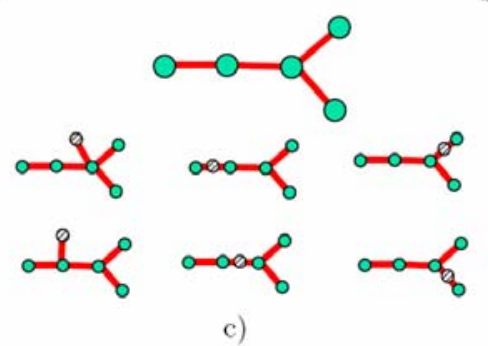

Fig. 2. Illustration of the simple "add node to a node" or "bisect an edge" graph grammar. a) We start with a simple 2-star from which one can generate three distinct graphs shown. The "Op1" operation is adding a node to a node, operations "Op1" and "Op2" are edge bisections (here they are topologically equivalent to adding a node to a terminal node of the initial 2-star). For illustration let us suppose that the "Op2" operation gives the biggest elastic energy decrement, thus it is the "optimal" operation. b) From the graph obtained one can generate 5 distinct graphs and choose the optimal one. c) The process is continued until a definite number of nodes are inserted.

Definition. Principal tree is an acyclic primitive elastic principal graph.

Definition. 'Add a node, bisect an edge' graph grammar $O^{(\text {grow })}$ applicable for the class of primitive elastic graphs consists of two operations: 1) The transformation "add a node" can be applied to any vertex $v$ of $G$ : add a new node $z$ and a new edge $(v, z) ; 2)$ The transformation "bisect an edge" is applicable to any pair of graph vertices $v, v^{\prime}$ connected by an edge $\left(v, v^{\prime}\right)$ : delete edge $\left(v, v^{\prime}\right)$, add a vertex $z$ and two edges, $(v, z)$ and $\left(z, v^{\prime}\right)$. The transformation of the elastic structure (change in the star list) is induced by the change of topology, because the elastic graph is primitive. Consecutive application of the operations from this grammar generates trees, i.e. graphs without cycles.

Definition. 'Remove a leaf, remove an edge' graph grammar $O^{(\text {shrink) }}$ applicable for the class of primitive elastic graphs consists of two operations: 1) The transformation 'remove a leaf' can be applied to any vertex $v$ of $G$ with connectivity degree equal to 1: remove $v$ and remove the edge $\left(v, v^{\prime}\right)$ connecting $\mathrm{V}$ to the tree; 2) The transformation 'remove an edge' is applicable to any pair of graph vertices $v, v^{\prime}$ connected by an edge $\left(v, v^{\prime}\right)$ : delete edge $\left(v, v^{\prime}\right)$, delete vertex $v$, merge the $k$-stars for which $v$ and $v^{\prime}$ are the central nodes and make a new $k$-star for which $v$ is the central node with a set of neighbors which is the union of the neighbors from the $k$-stars of $v$ and $v$ '.

Also we should define the structural complexity measure $\mathrm{SC}(G)=\mathrm{SC}\left(|V|,|E|,\left|S_{2}\right|, \ldots,\left|S_{\mathrm{m}}\right|\right)$. Its concrete form depends on the application field. Here are some simple examples: 
1) $\operatorname{SC}(G)=|V|$ : i.e., the graph is considered more complex if it has more vertices;

2) $\operatorname{SC}(G)=\left\{\begin{array}{c}\left|S_{3}\right|, \text { if }\left|S_{3}\right| \leq b_{\max } \text { and } \sum_{k=4}^{m}\left|S_{k}\right|=0 \\ \infty, \text { otherwise }\end{array}\right.$,

i.e., only $b_{\max }$ simple branches (3-stars) are allowed in the principal tree.

Using the sequence $\left\{O^{(\text {grow })}, O^{(\text {grow })}, O^{(\text {shrink })}\right\}$ in the above-described algorithm for estimating the elastic principal graph gives an approximation to the principal trees. Introducing the 'tree trimming' grammar $O^{(\text {shrink })}$ allows to produce principal trees closer to the global optimum, trimming excessive tree branching and fusing $k$-stars separated by small 'bridges'.

Principal trees can have applications in data visualization. A principal tree is embedded into a multidimensional data space. It approximates the data so that one can project points from the multidimensional space into the closest node of the tree. The tree by its construction is a one-dimensional object, so this projection performs dimension reduction of the multidimensional data. The question is how to produce a planar tree layout? Of course, there are many ways to layout a tree on a plane without edge intersection. But it would be useful if both local tree properties and global distance relations would be represented using the layout. We can require that

1) In a two-dimensional layout, all $k$-stars should be represented equiangular; this is the small penalty configuration;

2) The edge lengths should be proportional to their length in the multidimensional embedding; thus one can represent between-node distances.

This defines a tree layout up to global rotation and scaling and also up to changing the order of leaves in every $k$-star. We can change this order to eliminate edge intersections, but the result can not be guaranteed. In order to represent the global distance structure, it was found (Gorban et al., 2008) that a good approximation for the order of $k$-star leaves can be taken from the projection of every $k$-star on the linear principal plane calculated for all data points, or on the local principal plane in the vicinity of the $k$-star, calculated only for the points close to this star. The resulting layout can be further optimized using some greedy optimization methods.

The point projections are then represented as pie diagrams, where the size of the diagram reflects the number of points projected into the corresponding tree node. The sectors of the diagram allow us to show proportions of points of different classes projected into the node (see an example on Fig. 3). 


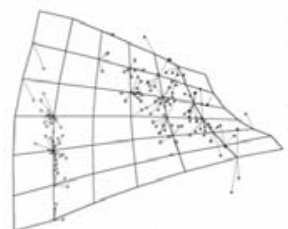

a)

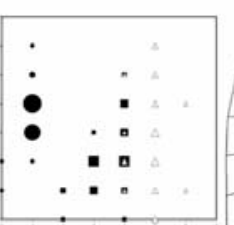

b)

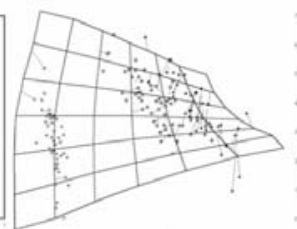

c)

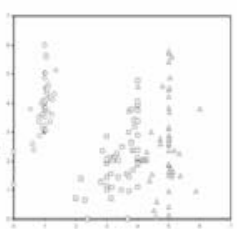

d)

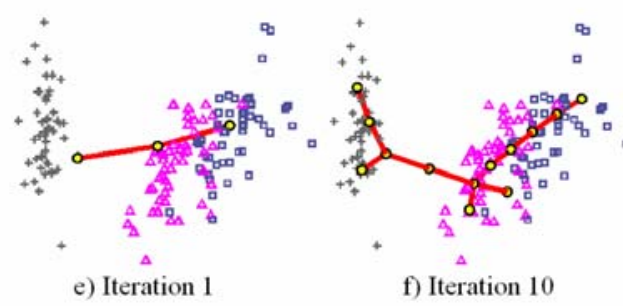

$+$
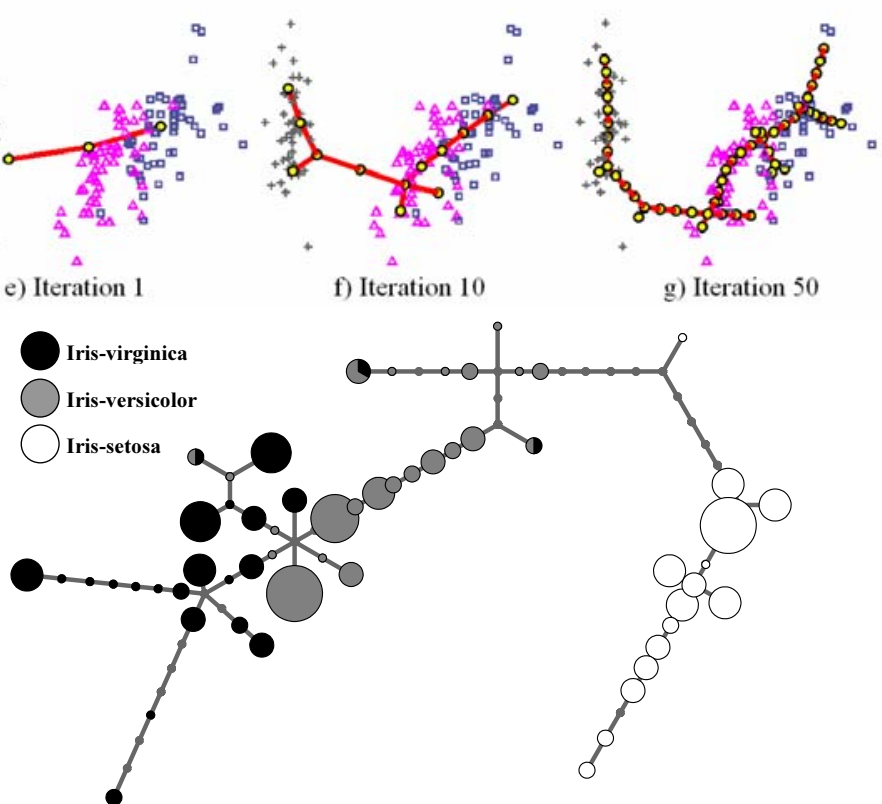

h)

Fig. 3. Principal manifold and principal tree for the Iris dataset. a) View of the principal manifold projected on the first two principal components, the data points are shown projected into the closest vertex of the elastic net; b) visualization of data points in the internal coordinates, here classes are represented in the form of Hinton diagrams: the size of the diagram is proportional to the number of points projected, the shape of the diagram denote three different point classes; c) same as a, but the data points are shown projected into the closest point of the piecewise linearly interpolated elastic map; d) same as d, but based on projection shown in c); e)-g) First 50 iterations of the principal tree algorithm, the tree is shown projected onto the principal plane; $h$ ) metro map representation of the Iris dataset.

This data display was called a "metro map" since it is a schematic and "idealized" representation of the tree and the data distribution with inevitable distortions made to produce a 2D layout. However, using this map one can still estimate the distance from a point (tree node) to a point passing through other points. This map is inherently unrooted (as a real metro map). It is useful to compare this metaphor with trees produced by hierarchical clustering where the metaphor is closer to a "genealogy tree".

\section{PrinCIPAL CUBiC COMPLEXES}

Elastic nets introduced above are characterized by their internal dimension $\operatorname{dim}(G)$. The way to generalize this characteristics on other elastic graphs is to utilize the notion of cubic complex (Gorban et al, 2007). 
Definition. Elastic cubic complex $K$ of internal dimension $r$ is a Cartesian product $G_{1} \times \ldots \times G_{r}$ of elastic graphs $G_{1}, \ldots G_{r}$. It has the vertex set $V_{1} \times \ldots \times V_{r}$. Let $1 \leq i \leq r$ and $v_{j} \in V_{j}(j \neq i)$. For this set of vertices, $\left\{v_{j}\right\}_{j \neq i}$, a copy of $G_{i}$ in $G_{1} \times \ldots \times G_{r}$ is defined with vertices $\left(v_{1}, \ldots, v_{i-1}, v, v_{i+1}, \ldots, v_{r}\right)\left(v \in V_{i}\right)$, edges

$\left(\left(v_{1}, \ldots, v_{i-1}, v, v_{i+1}, \ldots, v_{r}\right),\left(v_{1}, \ldots, v_{i-1}, v^{\prime}, v_{i+1}, \ldots, v_{r}\right)\right),\left(v, v^{\prime}\right) \in E_{i}$

and, similarly, $k$-stars of the form $\left(v_{1}, \ldots, v_{i-1}, S_{k}, v_{i+1}, \ldots, v_{r}\right)$, where $S_{k}$ is a $k$-star in $G_{i}$. For any $G_{i}$ there are $\prod_{j \neq i}\left|V_{j}\right|$ copies of $G_{i}$ in $G$. Sets of edges and $k$-stars for Cartesian product are unions of that set through all copies of all factors. A map $\varphi: V_{1} \times \ldots \times V_{r} \rightarrow \mathbf{R}^{m}$ maps all the copies of factors into $\mathbf{R}^{m}$ too.

Remark. By construction, the energy of the elastic graph product is the energy sum of all factor copies. It is, of course, a quadratic functional of $\phi$.

If we approximate multidimensional data by a $r$-dimensional object, the number of points (or, more generally, elements) in this object grows with $r$ exponentially. This is an obstacle for grammar-based algorithms even for modest $r$, because for analysis of the rule $A \rightarrow B$ applications we should investigate all isomorphic copies of $A$ in $G$. Introduction of a cubic complex is useful factorization of the principal object which allows to avoid this problem.

The only difference between the construction of general elastic graphs and factorized graphs is in the application of the transformations. For factorized graphs, we apply them to factors. This approach significantly reduces the amount of trials in selection of the optimal application. The simple grammar with two rules, "add a node to a node, or bisect an edge," is also powerful here, it produces products of primitive elastic trees. For such a product, the elastic structure is defined by the topology of the factors.

\section{INCOMPLETE DATA}

Some of the methods described above allow to use incomplete data in a natural way. Let us represent an incomplete observation by $\mathbf{x}=\left(x_{1}, \ldots, @, \ldots, @, \ldots, x_{m}\right)$, where the ‘@’ symbol denotes a missing value.

Definition. Scalar product between two incomplete observations $\mathbf{x}$ and $\mathbf{y}$ is $(\mathbf{x}, \mathbf{y})=\sum_{i \neq @}^{m} x_{i} y_{i}$. Then the Euclidean distance is $\sqrt{(\mathbf{x}-\mathbf{y})^{2}}=\sqrt{\sum_{i \neq @}^{m}\left(x_{i}-y_{i}\right)^{2}}$.

Remark. This definition has a very natural geometrical interpretation: an incomplete observation with $k$ missing values is represented by a $k$-dimensional linear manifold $L_{k}$, parallel to $k$ coordinate axes corresponding to the missing data.

Thus, any method which uses only scalar products or/and Euclidean distances can be applied for incomplete data with some minimal modifications subject to 
random and not too dense distribution of missing values in $X$. For example, the iterative method for SVD for incomplete data matrix was developed (Roweis (1998); Gorban \& Rossiev, 1999).

There are, of course, other approaches to incomplete data in unsupervised learning (for example, those presented by Little \& Rubin (1987)).

\section{IMPLICIT METHODS}

Most of the principal objects introduced in this paper are constructed as explicit geometrical objects embedded in $\mathbf{R}^{m}$ to which we can calculate the distance from any object in $X$. In this way, they generalize the "data approximation"-based (\#1) and the "variation-maximization"-based (\#2) definitions of linear PCA. There also exists the whole family of methods, which we only briefly mention here, that generalize the "distance distortion minimization" definition of PCA (\#3).

First, some methods take as input a pairwise distance (or, more generally, dissimilarity) matrix $D$ and construct such a configuration of points in a lowdimensional Euclidean space that the distance matrix $D$ ' in this space reproduce $D$ with maximal precision. The most fundamental in this series is the metric multidimensional scaling (Kruskal, 1964). The next is the Kernel PCA approach (Schölkopf et al., 1997) which takes advantage of the fact that for the linear PCA algorithm one needs only the matrix of pairwise scalar products (Gramm matrix) but not the explicit values of coordinates of $X$. It allows to apply the kernel trick (Aizerman et al., 1964) and substitute the Gramm matrix by the scalar products calculated with use of some kernel functions. Kernel PCA method is tightly related to the classical multidimensional scaling (Williams, 2002).

Local Linear Embedding or LLE (Roweis \& Saul, 2000) searches for such a $N \times N$ matrix $A$ that approximates given $\mathbf{x}^{i}$ by a linear combination of $n$ vectorsneighbours of $\mathbf{x}^{i}: \sum_{i=1}^{N}\left\|\mathbf{x}^{i}-\sum_{k=1}^{N} A_{k}^{i} \mathbf{x}^{k}\right\|^{2} \rightarrow \min$, where only such $A_{k}^{i} \neq 0$, if $k$ is one of the $n$ closest to $\mathbf{x}^{i}$ vectors. After one constructs such a configuration of points in $\mathbf{R}^{s}, s<<m$, that $\mathbf{y}^{i}=\sum_{k=1}^{N} A_{k}^{i} \mathbf{y}^{k}, \mathbf{y}^{i} \in \mathbf{R}^{s}$, for all $i=1 \ldots N$. The coordinates of such embedding are given by the eigenvectors of the matrix $(1-A)^{\mathrm{T}}(1-A)$.

ISOMAP (Tenenbaum et al., 2000) and Laplacian eigenmap (Belkin \& Niyogi, 2003; Nadler et al., 2008) methods start with construction of the neighbourhood graph, i.e. the graph in which close in some sense data points are connected by (weighted) edges. This weighted graph can be represented in the form of a weighted adjacency matrix $W=\left\{W_{i j}\right\}$. From this graph, ISOMAP constructs a new distance matrix $D^{(I S O M A P)}$, based on the path lengths between two points in the neighbourhood graph, and the multimensional scaling is applied to $D^{(I S O M A P)}$. Laplacian map solves the eigenproblem $L \mathbf{f}_{\lambda}=\lambda S \mathbf{f}_{\lambda}$, where 


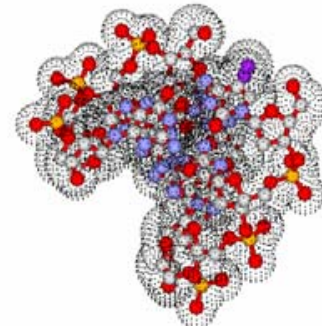

a)

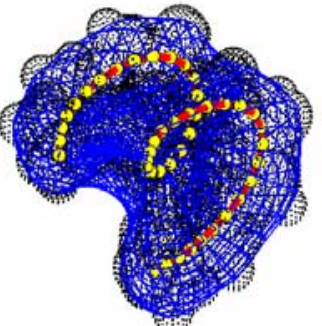

b)

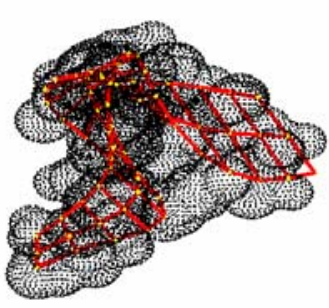

c)

Fig. 4. Principal objects approximating molecular surface of a short stretch of DNA molecule. a) stick-and-balls model of the DNA stretch and the initial molecular surface (black points); b) one- and two-dimensional spherical principal manifolds for the molecular surface; c) simple principal cubic complex (product of principal trees) which does not have any branching in this case.

$S=\operatorname{diag}\left\{\sum_{j=1}^{N} W_{0 j}, \cdots, \sum_{j=1}^{N} W_{N j}\right\}, L=S-W$ is the Laplacian matrix. The trivial constant solution corresponding to the smallest eigenvalue $\lambda_{0}=0$ is discarded, while the elements of the eigenvectors $\mathbf{f}_{\lambda_{1}}, \mathbf{f}_{\lambda_{2}}, \cdots, \mathbf{f}_{\lambda_{s}}$, where $\lambda_{1}<\lambda_{2}<\ldots<\lambda_{s}$, give the $s$-dimensional projection of $\mathbf{x}^{i}$, i.e. $P\left(\mathbf{x}^{i}\right)=\left\{\mathbf{f}_{\lambda_{1}}(i), \mathbf{f}_{\lambda_{2}}(i), \cdots, \mathbf{f}_{\lambda_{s}}(i)\right\}$.

Finally, one can implicitly construct projections into smaller dimensional spaces by training auto-associative neural networks with narrow hidden layer. An overview of the existing Neural PCA methods can be found in (Gorban et al, 2008).

\section{EXAMPLE: PRINCIPAL OBJECTS FOR THE IRIS DATASET}

On Fig. 3 we show application of the elastic principal manifolds and principal trees algorithms to the standard Iris dataset (Fisher, 1936). As expected, twodimensional approximation of the principal manifold in this case is close to the linear principal plane. One can also see that the principal tree illustrates well the fact of almost complete separation of classes in data space.

\section{EXAMPLE: PRINCIPAL OBJECTS FOR MOLECULAR SURFACES}

A molecular surface defines the effective region of space which is occupied by a molecule. For example, the Van-der-Waals molecular surface is formed by surrounding every atom in the molecule by a sphere of radius equal to the characteristic radius of the Van-der-Waals force. After all the interior points are eliminated, this forms a complicated non-smooth surface in 3D. In practice, this surface is sampled by a finite number of points.

Using principal manifolds methodology, we constructed a smooth approximation of such molecular surface for a small piece of a DNA molecule (several nucleotides long). First, we have made an approximation of this dataset by a 1D principal curve. Interestingly, this curve followed the backbone of the molecule, forming a spiral (see Fig. 4). Second, we approximated the molecular surface by a $2 \mathrm{D}$ manifold. The topology of the surface is expected to be spherical, so we applied spherical topology of the elastic net for optimisation. We should notice 
that since it is impossible to make the lengths of all edges equal for the spherical grid, corrections were performed for the edge elasticities during the grid initialization (shorter edges are given larger $\lambda_{i} \mathrm{~s}$ ). Third, we applied the method for constructing principal cubic complexes, namely, graph product of principal trees, which produced somewhat trivial construction (because no branching was energetically optimal): product of two short elastic principal curves, forming a double spirale.

\section{EXAMPLE: PRINCIPAL OBJECTS DECIPHER GENOME}

A dataset $X$ can be constructed for a string sequence using short word frequency dictionary approach in the following way: 1) the notion of word is defined; 2) the set of all possible short words is defined, let us say that we have $m$ of them; 3 ) a number $N$ of text fragments of certain width is sampled from the text; 4) in each fragment the frequency of occurrences of all possible short words is calculated and, thus, each fragment is represented as a vector in multidimensional space $\mathbf{R}^{m}$. The whole text then is represented as a dataset of $N$ vectors embedded in $\mathbf{R}^{m}$.

We systematically applied this approach to available bacterial genomic sequences. In our case we defined: 1) a word is a sequence of three letters from the $\{A, C, G, T\}$ alphabet (triplet); 2) evidently, there are 64 possible triplets in the $\{A, C, G, T\}$ alphabet; 3) we sampled 5000-10000 fragments of width 300 from a genomic sequence; 4) we calculated the frequencies of non-overlapping triplets for every fragment.

The constructed datasets are interesting objects for data-mining, because 1) they have a non-trivial cluster structure which usually contains various configurations of 7 clusters (see Fig. 5); 2) class labels can be assigned to points accordingly to available genome annotations; in our case we put information about presence (in one of six possible frameshifts) or absence of the coding information in the current position of a genome; 3) using data mining techniques here has immediate applications in the field of automatic gene recognition and in others, see, for example, (Carbone et al, 2003). On Fig. 5 we show application of both classical PCA and the metro map methods for several bacterial genomes. Look at http://www.ihes.fr/ zinovyev/7clusters web-site for further information. 


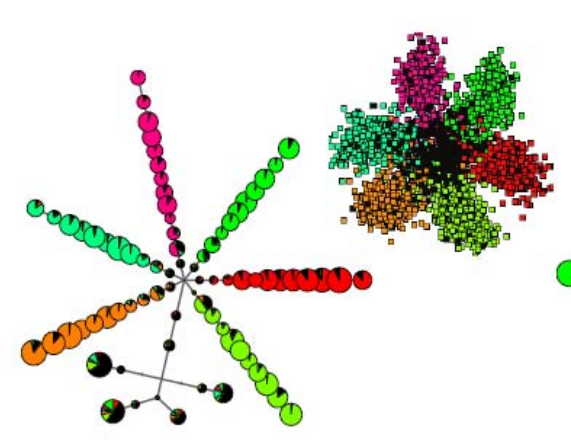

Streptomyces coelicolors

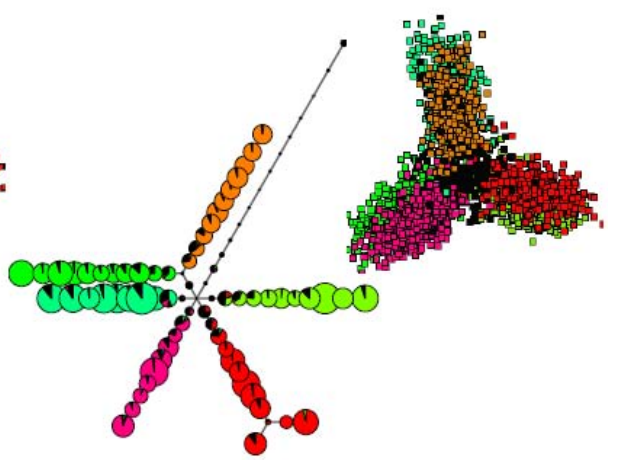

Fusobacterium nucleatum

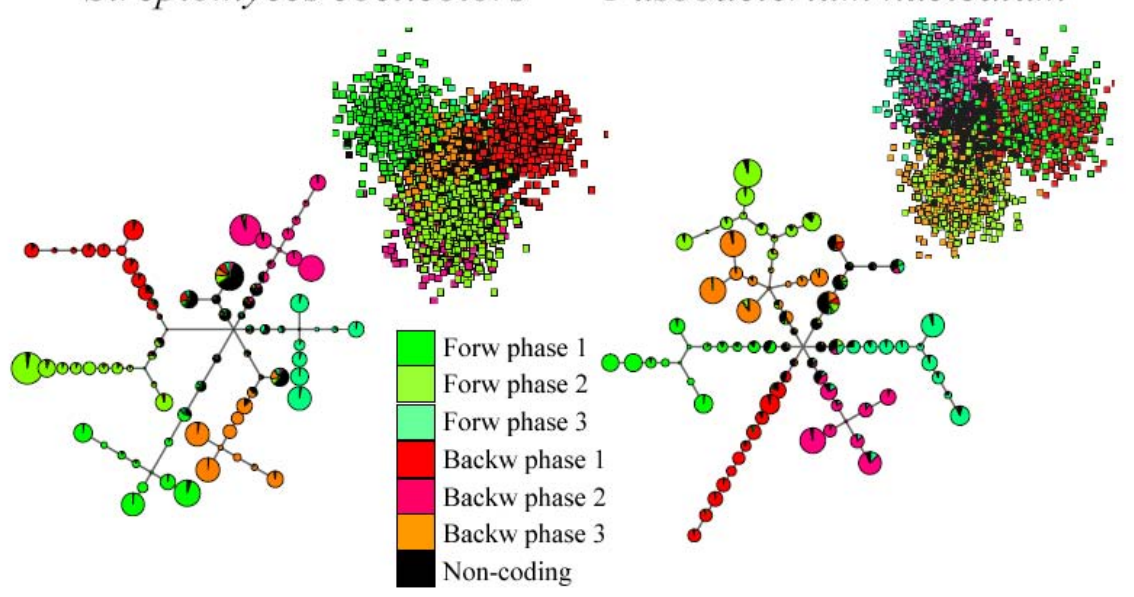

Bacillus halodurans

Fig. 5. Seven cluster structures presented for 4 selected genomes. A genome is represented as a collection of points (text fragments represented by their triplet frequencies) in the 64-multidimensional space. Color codes denote point classes corresponding to 6 possible frameshifts when a random fragment overlaps with a coding gene ( 3 in the forward and 3 in the backward direction of the gene), and the black color corresponds to non-coding regions. For every genome a principal tree ("metro map" layout) is shown together with 2D PCA projection of the data distribution. Note that the clusters that appear to be mixed on the PCA plot for Ercherichia coli (they remain mixed in 3D PCA as well) are well separated on the "metro map" which proves that they are well-separated in $\mathbf{R}^{64}$.

We provide these three examples only for illustrative purposes. More detailed examples of application of the principal graph and manifold methodology can be found in (Gorban et al., 2008).

\section{CONCLUSION}

In this chapter we gave a brief practical introduction into the methods of construction of principal objects, i.e. objects embedded in the 'middle' of the multidimensional data set. As a basis, we took the unifying framework of mean squared distance approximation of finite datasets which allowed to look at the principal graphs and manifolds as generalizations of the mean point notion. 


\section{REFERENCES}

1) Aizerman M., Braverman E., and Rozonoer L. (1964). Theoretical foundations of the potential function method in pattern recognition learning. Automation and Remote Control 25: 821-837.

2) Arthur D., Vassilvitskii S. (2007). K-means++ The Advantages of Careful Seeding. In: Proceedings of the Eighteenth Annual ACM-SIAM Symposium on Discrete Algorithms, SODA 2007 (pp. 1027-1035). New Orleans, Louisiana, USA, January 7-9.

3) Aluja-Banet, T. and Nonell-Torrent, R. (1991). Local principal component analysis. Qüestioó 3, 267-278.

4) Bau III D., Trefethen L.N. (1997). Numerical linear algebra. SIAM Press, Philadelphia. (Lecture 31)

5) Bauer F. L. (1957). Das verfahren der Treppeniteration und verwandte verfahren zur losung algebraischer eigenwert probleme, Z. Angew Math. Phys. 8, 214-235.

6) Belkin M. and Niyogi P. (2006). Laplacian Eigenmaps for Dimensionality Reduction and Data Representation. Neural Computation 15(6): 1373-1396.

7) Bishop C.M., Svensén M., and Williams C.K.I. (1998). GTM: The generative topographic mapping. Neural Computation 10(1), 215-234

8) Braverman E.M. (1970). Methods of extremal grouping of parameters and problem of apportionment of essential factors. Automation and Remote Control (1), 108-116

9) Cochran, R. N., Horne F. H. (1977). Statistically weighted principal component analysis of rapid scanning wavelength kinetics experiments. Anal. Chem. 49, 846-853.

10) Delicado P. (2001). Another look at principal curves and surfaces. Journal of Multivariate Analysis 77, 84-116

11) Dempster A., Laird N., and Rubin D. (1977). Maximum likelihood from incomplete data via the EM algorithm. Journal of the Royal Statistical Society, Series B, 39(1):1-38.

12) Diday E. (1979). Optimisation en classification automatique, Tome 1,2. INRIA, Rocquencourt (in French)

13) Durbin R., Willshaw D. (1987). An analogue approach to the travelling salesman problem using an elastic net method. Nature 326, $689-691$.

14) Efron, B. (1967). The two sample problem with censored data. Proc. Fifth Berkeley Simp. Math. Statist. Probab. 4, 831-853. Univ.California Press, Berkeley.

15) Einbeck, J., Tutz, G., and Evers, L. (2005). Local principal curves. Statistics and Computing 15, 301-313.

16) Einbeck J., Evers L., and Bailer-Jones C. (2008). Representing Complex Data Using Localized Principal Components With Application To Astronomical Data. In Gorban A., Kégl B., Wunch D., Zinovyev A. (Ed.) Principal Manifolds for Data Visualization and Dimension Reduction, Lecture Notes in Computational Science and Engineering 58 (pp. 178-201). Springer, Berlin-Heidelberg.

17) Erwin E., Obermayer K., and Schulten K. (1992). Self-organizing maps: ordering, convergence properties and energy functions. Biological Cybernetics 67, 47-55. 
18) Fisher R.A. (1936). The Use of Multiple Measurements in Taxonomic Problems. Annals of Eugenics 7, 179-188.

19) Flury B. (1990). Principal points. Biometrika, 77, 33-41.

20) Fréchet, M. (1948). Les élements aléatoires de nature quelconque dans un espace distancié. Ann. Inst. H. Poincaré 10, 215-310.

21) Fukunaga K., Olsen D. (1971). An algorithm for finding intrinsic dimensionality of data. IEEE Transactions on Computers 20 (2), 176-183

22) Gabriel K. R. and Zamir S. (1979), Lower rank approximation of matrices by least squares with any choices of weights, Technometrics, 21, pp. 298489.

23) Gorban A.N., Rossiev A.A.. (1999). Neural network iterative method of principal curves for data with gaps. J Comput Sys Sc Int 38 (5), 825-830.

24) Gorban A.N., Pitenko A.A., Zinov'ev A.Y., Wunsch D.C. (2001). Vizualization of any data using elastic map method. Smart Engineering System Design 11, 363-368.

25) Gorban, A.N., Zinovyev, A.Yu., and Wunsch, D.C. (2003). Application of the method of elastic maps in analysis of genetic texts. In Proceedings of International Joint Conference on Neural Networks. IJCNN Portland, Oregon, July 20-24.

26) Gorban A., Zinovyev A. (2005). Elastic Principal Graphs and Manifolds and their Practical Applications. Computing 75,359 -379

27) Gorban A., Sumner N., Zinovyev A. (2007). Topological grammars for data approximation. Applied Mathematics Letters 20(4), 382-386.

28) Gorban A., Kégl B., Wunch D., Zinovyev A. (Ed.) (2008). Principal Manifolds for Data Visualization and Dimension Reduction. Lecture Notes in Computational Science and Engineering, Vol. 58: Berlin-Heidelberg, Springer.

29) Gorban A., Sumner N.R., Zinovyev A. (2008). Beyond The Concept of Manifolds: Principal Trees, Metro Maps, and Elastic Cubic Complexes. In Gorban A., Kégl B., Wunch D., Zinovyev A. (Ed.) Principal Manifolds for Data Visualization and Dimension Reduction, Lecture Notes in Computational Science and Engineering 58 (pp. 96-130). Springer, BerlinHeidelberg.

30) Gorban A., Zinovyev A. (2008). Elastic Maps and Nets for Approximating Principal Manifolds and Their Application to Microarray Data Visualization. In Gorban A., Kégl B., Wunch D., Zinovyev A. (Ed.) Principal Manifolds for Data Visualization and Dimension Reduction, Lecture Notes in Computational Science and Engineering 58 (pp. 96-130). Springer, Berlin-Heidelberg.

31) Gorban A., Zinovyev A. (2008). Elastic Maps and Nets for Approximating Principal Manifolds and Their Application to Microarray Data Visualization. In Gorban A., Kégl B., Wunch D., Zinovyev A. (Ed.) Principal Manifolds for Data Visualization and Dimension Reduction, Lecture Notes in Computational Science and Engineering 58 (pp. 96-130). Springer, Berlin-Heidelberg.

32) Hartigan, J.A. (1975). Clustering Algorithms. Wiley, New-York.

33) Hastie T. (1984). Principal Curves and Surfaces. PhD Thesis, Stanford University, California.

34) Hotelling H. (1933). Analisys of a complex of statistical variables into principal components. Journal of Educational Psychology 24, 417-441. 
35) Jolliffe I.T. (2002). Principal Component Analysis, Series: Springer Series in Statistics, 2nd ed., XXIX. Springer, NY.

36) Kambhatla, N. and Leen, T. K. (1997). Dimension reduction by local PCA. Neural Computation 9, 1493-1516

37) Karhunen K. (1946). Zur Spektraltheorie Stochastischer Prozesse. Ann.Acad. Sci. Fennicae, 37.

38) Kégl B. (1999). Principal curves: learning, design, and applications. Ph.D. Thesis, Concordia University, Canada.

39) Kégl B., Krzyzak A. (2002). Piecewise linear skeletonization using principal curves. IEEE Transactions on Pattern Analysis and Machine Intelligence 24(1), 59-74.

40) Kohonen, T. (1982). Self-organized formation of topologically correct feature maps. Biological Cybernetics 43, 59-69

41) Kohonen T. (1997). Self-Organizing Maps. Springer: Berlin - Heidelberg.

42) Koren Y., \& Carmel L. (2004). Robust linear dimensionality reduction. IEEE Transactions on Visualisation and Computer Graphics, 10 (4), $459-470$.

43) Kruskal J.B. (1964). Multidimensional scaling by optimizing goodness of fit to a nonmetric hypothesis. Psychometrika 29: 1-27.

44) Little, R.J.A. and Rubin, D.B. (1987). Statistical Analysis with Missing Data. New York: John Wiley.

45) Liu Z.-Y., Chiu K.-C., and Xu L. (2003). Improved system for object detection and star/galaxy classification via local subspace analysis. Neural Networks 16, 437-451

46) Lloyd S. (1957). Least square quantization in PCM's. Bell Telephone Laboratories Paper.

47) Loève M.M. (1955). Probability Theory. Princeton, N.J.: VanNostrand.

48) Löwe, M. (1993). Algebraic approach to single-pushout graph transformation. Theor. Comp. Sci. 109, 181-224.

49) Lumley J.L. (1967). The Structure of Inhomogeneous Turbulent Flows. In Yaglom A.M., Tatarski V.I (Eds.) Atmospheric turbulence and radio propagation (pp. 166-178). Moscow: Nauka.

50) MacQueen J. (1967). Some methods for classification and analysis of multivariate observations. In Proc. 5th Berkeley Symp. on Math. Statistics and Probability, pages 281-297.

51) Mirkin, B. (2005). Clustering for Data Mining: A Data Recovery Approach. Chapman and Hall, Boca Raton.

52) Mulier F., Cherkassky V. (1995). Self-organization as an iterative kernel smoothing process. Neural Computation 7, 1165-1177.

53) Nadler B., Lafon S., Coifman R., Kevrekidis I. G. (2008). Diffusion Maps a Probabilistic Interpretation for Spectral Embedding and Clustering Algorithms. In Gorban A., Kégl B., Wunch D., Zinovyev A. (Ed.) Principal Manifolds for Data Visualization and Dimension Reduction, Lecture Notes in Computational Science and Engineering 58 (pp. 69-96). Springer, BerlinHeidelberg.

54) Nagl, M. (1976). Formal languages of labelled graphs. Computing 16, $113-$ 137.

55) Ostrovsky R., Rabani Y., Schulman L.J., Swamy C. (2006). The Effectiveness of Lloyd-Type Methods for the k-Means Problem. In Proceedings of the 47th Annual IEEE Symposium on Foundations of 
Computer Science (pp. 165-176). IEEE Computer Society, Washington, DC, USA.

56) Pearson K. (1901). On lines and planes of closest fit to systems of points in space. Philosophical Magazine, series 6 (2), 559-572

57) Pelleg D., Moore A. (1999). Accelerating Exact $k$-means Algorithms with Geometric Reasoning. Proceedings of the Fifth International Conference on Knowledge Discovery in Databases, 277-281. AAAI Press

58) Ritter H., Martinetz T. and Schulten K. (1992). Neural Computation and Self-Organizing Maps: An Introduction. Addison-Wesley Reading, Massachusetts.

59) Roweis S. (1998). EM algorithms for PCA and SPCA. In Advances in Neural Information Processing Systems (pp. 626-632). MIT Press, Cambridge, MA (USA).

60) Roweis S., Saul L. (2000). Nonlinear dimensionality reduction by locally linear embedding. Science 290: 2323-2326.

61) Schölkopf B., Smola A.J., Müller K.-R. (1997). Kernel Principal Component Analysis. ICANN: 583-588.

62) Smola A.J., Mika S., Schölkopf B., Williamson R.C. (2001). Regularized Principal Manifolds. Journal of Machine Learning Research 1: 179-209.

63) Steinhaus H. (1956). Sur la division des corps materiels en parties. Bull. Acad. Polon. Sci., C1. III vol IV: 801-804.

64) Strang G. (1993). The Fundamental Theorem of Linear Algebra. The American Mathematical Monthly 100 (9), pp. 848-855

65) Sylvester J.J. (1889). On the reduction of a bilinear quantic of the $n$th order to the form of a sum of $\mathrm{n}$ products by a double orthogonal substitution. Messenger of Mathematics 19, 42-46.

66) Tarpey T., \& Flury B. (1996). Self-consistency: a fundamental concept in statistics. Statistical Science, 11(3), 229-243.

67) Tenenbaum J. B., de Silva V. and Langford J. C. (2000). A global geometric framework for nonlinear dimensionality reduction. Science 290: 2319-2323.

68) Tibshirani R. (1992). Principal curves revisited. Statistics and Computation 2, 183-190.

69) Verbeek J. J., Vlassis N., Kröse B. (2002). A $k$-segments algorithm for finding principal curves. Pattern Recognition Letters 23 (8), 1009-1017.

70) Williams C.K.I. (2002). On a connection between Kernel PCA and metric multidimensional scaling. Machine Learning 46, 11-19.

71) Xu R., Wunsch D. (2008). Clustering, IEEE Press Series on Computational Intelligence, John Wiley \& Sons, NY,

72) Yin H. (2008). Learning Nonlinear Principal Manifolds by Self-Organising Maps, In Gorban A., Kégl B., Wunch D., Zinovyev A. (Ed.) Principal Manifolds for Data Visualization and Dimension Reduction, Lecture Notes in Computational Science and Engineering 58 (pp. 69-96). Springer, BerlinHeidelberg.

73) Zou H., Hastie T. (2005). Regularization and variable selection via the elastic net. J. R. Statist. Soc. B 67, Part 2, 301-320. 


\section{BIOGRAPHY}

Prof. Dr. Alexander Gorban obtained his PhD degree in differential equations and mathematical physics in 1980; and Dr. Sc. degree in biophysics in 1990. He holds now a chair of Applied mathematics at the University of Leicester, UK, and he is the Chief Scientist at the Institute for Computational Modelling Russian Academy of Sciences, Krasnoyarsk, Russia. His scientific interest include interdisciplinary problem of model reduction, topological dynamics, physical and chemical kinetics, mathematical biology and data mining.

Dr. Andrei Zinovyev obtained his university formation in theoretical physics. In 2001 he obtained his PhD degree in Computer Science working at the Institute of Computational Modeling of Russian Academy of Science, Siberian Branch (Krasnoyarsk, Russia). After defending his $\mathrm{PhD}$, he moved to France, at Institut des Hautes Etudes Scientifiques in Bures-sur-Yvette to work as a post-doc during 3 years in the mathematical biology group of Professor Misha Gromov. Since January 2005, he is the head of the computational systems biology of cancer team at the Bioinformatics Service of Institut Curie in Paris. His main area of scientific expertise is bioinformatics, systems biology of cancer, dimension reduction in high-throughput data analysis and model reduction in the dynamical models.

\section{KEY TERMS AND THEIR DEFINITIONS}

Principal components: such an orthonormal basis in which the covariance matrix is diagonal.

Principal manifold: intuitively, a smooth manifold going through the middle of data cloud; formally, there exist several definitions for the case of data distributions: 1) Hastie and Stuelze's principal manifolds are self-consistent curves and surfaces; 2) Kegl's principal curves provide the minimal mean squared error given the limited curve length; 3) Tibshirani's principal curves maximize the likelihood of the additive noise data model; 4) Smola's regularized principal manifolds minimize some form of a regularized quantization error functional; and some other definitions.

Principal graph: a graph embedded in the multidimensional data space, providing the minimal mean squared distance to the dataset together with deviation from an "ideal" configuration (for example, from pluriharmonic graph) and not exceeding some limits on complexity (in terms of the number of structural elements and the number of graph grammar transformations needed for obtaining the principal graph from some minimal graph).

Self-consistent approximation: approximation of a dataset by a set of vectors such that every point $\mathbf{y}$ in the vector set is a conditional mean of all points from the dataset that are projected in $\mathbf{y}$.

Expectation/Maximisation algorithm: generic splitting algorithmic scheme with use of which almost all algorithms for estimating principal objects are constructed; it consists of two basic steps: 1) projection step, at which the data is projected onto the approximator, and 2) maximization step, at which the approximator is optimized given the projections obtained at the previous step. 